\title{
Relative derived equivalences and relative homological dimensions
}

\author{
SHENGYONG PAN ${ }^{1,2}$ \\ ${ }^{1}$ Department of Mathematics, Beijing Jiaotong University, Beijing 100044, People's Republic of China \\ ${ }^{2}$ School of Mathematical Sciences, Beijing Normal University, Beijing 100875, People's Republic of China \\ E-mail:shypan@bjtu.edu.cn
}

\begin{abstract}
Let $\mathscr{A}$ be a small abelian category. For a closed subbifunctor $F$ of $\operatorname{Ext}_{\mathscr{A}}^{1}(-,-)$, Buan has generalized the construction of the Verdier's quotient category to get a relative derived category, where he localized with respect to $F$-acyclic complexes. In this paper, the homological properties of relative derived categories are discussed, and the relation with derived categories is given. For Artin algebras, using relatively derived categories, we give a relative version on derived equivalences induced by $F$-tilting complexes. We discuss the relationships between relative homological dimensions and relative derived equivalences.
\end{abstract}

2000 AMS Classification: 18E30,16G10;16S10,18G15.

Key words: relative derived category, $F$-tilting complex, relative derived equivalence, relative homological dimension.

\section{Introduction}

Hochschild [25] introduced relative homological algebra in categories of modules. And later, Heller, Butler and Horrocks developed it in more general categories with a relative abelian structure. Auslander and Solberg [2, 3, 4, 5, 6] applied relative homological algebra to the representation theory of Artin algebra. They studied relative homology in terms of subbifunctors of the functor $\operatorname{Ext}^{1}(-,-)$ and developed the general theory of relative cotilting modules for Artin algebras.

Derived categories were invented by Grothendieck-Verdier [45] in the early sixties. Today, they have widely been used in many branches: algebraic geometry, stable homotopy theory, representation theory, etc. In representation theory of Artin algebras, it is of interest to investigate whether two Artin algebras have equivalent derived categories. As is known, a Morita theory for derived equivalences was established by Rickard [40]. According to his theorem, two Artin algebras $A$ and $B$ are derived equivalent if and only if there is a tilting complex for $A$ such that $B$ is isomorphic to the endomorphism algebra of this complex. An interesting thing is to construct a new derived equivalence from a given one by finding a tilting complex. Rickard [41, 42] got new derived equivalence by tensor products and trivial extensions. In the recent years, Hu and $\mathrm{Xi}[28,30]$ have provided various techniques to construct new derived equivalences by finding tilting complexes. Another interesting thing is to find some invariance under derived equivalences. Derived equivalences between finite dimensional algebras over a field have many invariants. For instance, finiteness of finitistic dimension [39], Hochschild homology and cohomology [42] and $K$-theory [15] have been shown to be invariant under derived equivalences.

Derived categories have been used effectively in relative homological algebra. The main idea of relative homological algebra is to replace projective modules by relative projective modules. It is natural to study the corresponding version of the derived category in this context. Since then, the relative derived categories and relative tilting theory of Artin algebras have been extensively studied. Recently, Gao and Zhang [17] used Gorenstein homological algebra to get Gorenstein derived categories. Buan [7] also studied relative derived categories by localizing relative quasi-isomorphisms. Using the notion of relative derived categories, he generalized Happel's result on derived equivalences induced by tilting module to the relative setting. Is it very likely that relative tiltings provide something called relative derived equivalences? 
Motivated by Buan's work, we introduce relative derived equivalences for Artin algebras in this paper. A 'Morita' theory for relative derived categories is built, and some invariance of relative derived equivalences is founded. The aim of this paper is to discuss the relationships between relative derived equivalences and relative homological dimensions.

To describe the main result, it is convenient to fix some notations. Let $\Lambda$ and $\Gamma$ be Artin algebras. Assume that $F$ is a subbifunctor of $\operatorname{End}_{\Lambda}^{1}(-,-)$ which is of finite type. Denote by $\operatorname{gl} \cdot \operatorname{dim}(\Lambda)$ and fin.dim $(\Lambda)$ the global and the finitistic dimensions of $\Lambda$, respectively. Denote by $\operatorname{gl\cdot } \operatorname{dim}_{F}(\Lambda)$ and $\operatorname{fin} \cdot \operatorname{dim}_{F}(\Lambda)$ the $F$-global and the $F$-finitistic dimensions of $\Lambda$, respectively. Let $n \geq 0$ be an integer. If the complex $X^{\bullet}$ has the form:

$$
\cdots \rightarrow X^{-n} \rightarrow X^{-n+1} \rightarrow \cdots \rightarrow X^{-1} \rightarrow X^{0} \rightarrow \cdots,
$$

with $X^{i} \neq 0$ and the differential being radical map for $-n \leq i \leq 0$, then $n$ is called the term length of $X^{\bullet}$, denoted by $t\left(X^{\bullet}\right)$.

Our main result can be stated as follows:

Theorem (see Theorem 7.3) Let $L: \mathrm{D}_{\mathrm{F}}^{\mathrm{b}}(\Lambda) \rightarrow \mathrm{D}^{\mathrm{b}}(\Gamma)$ be a relative derived equivalence. Suppose $T^{\bullet}$ is an $F$-tilting complex for $\Lambda$ associated to $L$. Then

(1) $\operatorname{gl} \cdot \operatorname{dim}_{F}(\Lambda)-t\left(T^{\bullet}\right) \leq \operatorname{gl} \cdot \operatorname{dim}(\Gamma) \leq \operatorname{gl} \cdot \operatorname{dim}_{F}(\Lambda)+t\left(T^{\bullet}\right)+2$.

(2) fin. $\operatorname{dim}_{F}(\Lambda)-t\left(T^{\bullet}\right) \leq$ fin.dim $(\Gamma) \leq$ fin.dim $\operatorname{dim}_{F}(\Lambda)+t\left(T^{\bullet}\right)+2$.

We give the upper and lower bounds of $\operatorname{gl} \operatorname{dim}(\Gamma)$ (resp. fin.dim $(\Gamma)$ ) in the term length of relative tilting complex and gl.dim$F(\Lambda)$ (resp. fin. $\operatorname{dim}_{F}(\Lambda)$ ). In this theorem, if $F=\operatorname{Ext}_{\Lambda}^{1}(-,-)$, then $T^{\bullet}$ is a tilting complex for $\Lambda$ such that $\operatorname{End}\left(T^{\bullet}\right) \simeq \Gamma$. In the proof of this theorem, we infer that $\operatorname{gl} \operatorname{dim}(\Lambda)$ (resp. fin.dim $(\Lambda)$ ) and $\operatorname{gl} \cdot \operatorname{dim}(\Gamma)($ resp. fin.dim $(\Gamma))$ satisfy a similar formula but not the same as in this theorem, namely

(1) gl.dim $(\Lambda)-t\left(T^{\bullet}\right) \leq$ gl.dim $(\Gamma) \leq$ gl.dim $(\Lambda)+t\left(T^{\bullet}\right)$.

(2) fin.dim $(\Lambda)-t\left(T^{\bullet}\right) \leq$ fin.dim $(\Gamma) \leq$ fin.dim $(\Lambda)+t\left(T^{\bullet}\right)$.

This paper is organized as follows. In Section 2, we consider closed subbifunctors $F$ of $^{\operatorname{Ext}_{\mathscr{A}}}{ }^{1}(-,-)$, where $\mathscr{A}$ is a small abelian category. We introduce the notion of the relative derived category of $\mathscr{A}$ which was defined in [7]. The homological properties of relative derived categories are discussed, and the relation with derived categories is given in Section 3. In Section 4, we give the triangulated structure in the relative derived category. In Section 5, we show that, the quotient of the bounded relative derived category by the bounded homotopy category of relative projective complexes is equivalent to the relative stable category as triangulated categories. In Section 6, we introduce the notion of relative derived equivalences for Artin algebras. In Section 7, we prove the main result.

\section{Relative derived categories for abelian categories}

Let us explain the notion of relative derived categories. The notion of relative derived categories was introduced earlier by Generalov [19].

Let $\mathscr{A}$ be an abelian category. Suppose $A, C \in \mathscr{A}$. Denote by $\operatorname{Ext}_{\mathscr{A}}^{1}(C, A)$ the set of all exact sequences $0 \rightarrow A \rightarrow B \rightarrow C \rightarrow 0$ in $\mathscr{A}$ modulo the equivalence relation which is defined in the following usual way. Two exact sequences are equivalent if the following commutative diagram is commutative.

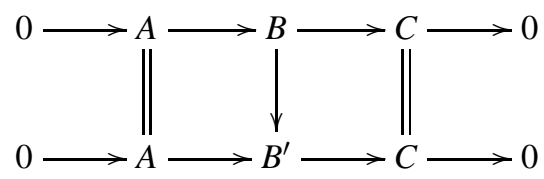

Since that any additive category has finite direct sums and in particular uniquely defined diagonal and codiagonal maps and that an abelian category has pullback and pushout pairs, it follows that $\operatorname{Ext}_{\mathscr{A}}^{1}(C, A)$ becomes an abelian group under Baer sum. Therefore, $\operatorname{Ext}_{\mathscr{A}}^{1}(-,-)$ defines an additive bifunctor $\mathscr{A}^{o p} \times$ $\mathscr{A} \longrightarrow \mathbf{A b}$, where $\mathbf{A b}$ is the category of abelian groups.

Consider additive non-zero subbifunctors $F$ of $\operatorname{Ext}^{1}(-,-)$. To each subfunctor corresponds a class of short exact sequences which are called $F$-exact sequences. The class of $F$-exact sequences is closed under 
the operations of pushout, pullback, Baer sums and direct sums (see [2] or [14]). Given a subbifunctor $F$ of $\operatorname{Ext}^{1}(-,-)$, we say that an exact sequence

$$
\eta: 0 \rightarrow X \rightarrow Y \rightarrow Z \rightarrow 0
$$

in $\mathscr{A}$ is an $F$-exact sequence if $\eta$ is in $F(Z, X)$. If $0 \rightarrow X \stackrel{f}{\rightarrow} Y \stackrel{g}{\rightarrow} Z \rightarrow 0$ is an $F$-exact sequence, then $f$ is called an $F$-monomorphism and $g$ is called an $F$-epimorphism. An object $P$ is said to be $F$-projective if for each $F$-exact sequence $0 \rightarrow X \rightarrow Y \rightarrow Z \rightarrow 0$, the sequence

$$
0 \rightarrow \mathscr{A}(P, X) \rightarrow \mathscr{A}(P, Y) \rightarrow \mathscr{A}(P, Z) \rightarrow 0
$$

is exact. An object $I$ is called $F$-injective if for each $F$-exact sequence $0 \rightarrow X \rightarrow Y \rightarrow Z \rightarrow 0$, the sequence

$$
0 \rightarrow \mathscr{A}(X, I) \rightarrow \mathscr{A}(Y, I) \rightarrow \mathscr{A}(Z, I) \rightarrow 0
$$

is exact. The subcategory of $\mathscr{A}$ consisting of all $F$-projective (resp. $F$-injective) modules is denoted by $\mathcal{P}(F)$ (resp. $I(F)$ ).

We have the following characterization of when subbifunctors of $\operatorname{Ext}^{1}(-,-)$ have enough projectives or injectives.

Lemma 2.1. 2, Theorem 1.12] Let $F$ be a subbifunctor of $\operatorname{Ext}^{1}(-,-)$.

(1) $F$ has enough projectives if and only if $F=F_{\mathcal{P}(F)}$ and $\mathcal{P}(F)$ is contravariantly finite in $\Lambda$-mod.

(2) $F$ has enough injectives if and only if $F=F^{I(F)}$ and $I(F)$ is covariantly finite in $\Lambda$-mod.

(3) If there is a finite number of indecomposable relative projectives (injectives) up to isomorphism, then there is also a finite number of relative injectives (projectives), and these numbers are the same.

Recall from [7, 14] that an additive subbifunctor $F$ is said to be closed if the following equivalence statements hold.

1) The composition of $F$-epimorphisms is an $F$-epimorphism.

2) The composition of $F$-monomorphisms is an $F$-monomorphism.

3 ) For each object $X$ the functor $F(X,-)$ is half exact on $F$-exact sequences.

4) For each object $X$ the functor $F(-, X)$ is half exact on $F$-exact sequences.

$5)$ The category $\mathscr{A}$ with respect to the $F$-exact sequences is an exact category.

We will give some basic examples of closed subbifunctors. Let $X$ be a full subcategory of $\mathscr{A}$ and for each pair of objects $A$ and $C$ in $\mathscr{A}$, we define

$$
F_{X}(C, A)=\{0 \rightarrow A \rightarrow B \rightarrow C \rightarrow 0 \mid(X, B) \rightarrow(X, C) \rightarrow 0 \text { is exact }\} .
$$

Dually we define for each pair of objects $A$ and $C$ in $\mathscr{A}$

$$
F^{X}(C, A)=\{0 \rightarrow A \rightarrow B \rightarrow C \rightarrow 0 \mid(B, X) \rightarrow(A, X) \rightarrow 0 \text { is exact }\} .
$$

Lemma 2.2. 14, Proposition 1.7] The additive subbifunctors $F_{X}$ and $F^{X}$ of $\operatorname{Ext}_{\mathscr{A}}^{1}(-,-)$ are closed for any subcategory $x$ of $\mathscr{A}$.

Remark. By Lemma 2.1 and Lemma 2.2, it follows that if a subbifunctor $F$ has enough projectives or injectives, then $F$ is a closed subfunctor.

Let $\mathrm{K}(\mathscr{A})$ be the homotopy category of complexes over $\mathscr{A}$. We denote by [1] the shift functor. A complex $X^{\bullet}$ with differential $d_{X}^{\bullet}$ is said to be $F$-acyclic if for each $i$ the induced complex

$$
0 \rightarrow \text { Imd }^{i-1} \rightarrow X^{i} \rightarrow \text { Imd }^{i} \rightarrow 0
$$

is an $F$-exact sequence. A map $h$ in $\mathrm{K}(\mathscr{A})$ is called an $F$-quasi-isomorphism if the mapping cone $M(h)$ is an $F$-acyclic complex. If $F$ has enough projectives, then $F$-exact sequences are exact sequences and $F$-acyclic complexes are acyclic complexes. But the converse is not true.

If the class of $F$-acyclic complexes are closed under the operation of mapping cone, then we call $F$ a triangulated subbifunctor. In this case the class of $F$-acyclic complexes is a null-system in $\mathrm{K}(\mathscr{A})$. Since the class of $F$-quasi-isomorphisms is a multiplicative system, it follows that we localize with respect to this system. 
Lemma 2.3. 7, Theorem 2.4] A subbifunctor is triangulated if and only if it is closed.

Now we assume that $F$ is a closed subbifunctor. It is clear that

$$
\mathscr{N}=\left\{X^{\bullet} \in \operatorname{Obj}(\mathrm{K}(\mathscr{A})) \mid X^{\bullet} \text { is an F-acyclic complex }\right\}
$$

is a null system and a thick subcategory of $\mathrm{K}(\mathscr{A})$. Define a morphism set

$$
\begin{array}{r}
\Sigma(\mathscr{N})=\left\{X^{\bullet} \stackrel{f^{\bullet}}{\rightarrow} Y^{\bullet} \mid \text { such that } X^{\bullet} \stackrel{f^{\bullet}}{\rightarrow} Y^{\bullet} \rightarrow Z^{\bullet} \rightarrow X^{\bullet}[1]\right. \text { is a distinguished triangle in } \\
\left.\mathrm{K}(\mathscr{A}) \text { with } Z^{\bullet} \in \mathscr{N}\right\} .
\end{array}
$$

The relative derived category of $\mathscr{A}$ is defined to be the Verdier quotient, that is, $\mathrm{D}_{\mathrm{F}}^{\mathrm{b}}(\mathscr{A}):=\mathrm{K}(\mathscr{A}) / \mathscr{N}=$ $\Sigma(\mathscr{N})^{-1} \mathrm{~K}(\mathscr{A})$.

The objects of $\mathrm{D}_{\mathrm{F}}^{\mathrm{b}}(\Lambda)$ are the same as for $\mathrm{K}(\mathscr{A})$. A map in $\mathrm{D}_{\mathrm{F}}^{\mathrm{b}}(\Lambda)$ from $X^{\bullet}$ to $Y^{\bullet}$ is the equivalence class of "roofs", that is, of fractions $g^{\bullet} / f^{\bullet}$ of the form $X^{\bullet} \stackrel{f^{\bullet}}{\longleftarrow} Z^{\bullet} \stackrel{g^{\bullet}}{\longrightarrow} Y^{\bullet}$, where $Z^{\bullet} \in \mathrm{K}(\mathscr{A})$, $f^{\bullet}: Z^{\bullet} \rightarrow X^{\bullet}$ is an $F$-quasi-isomorphism, and $g^{\bullet}: Z^{\bullet} \rightarrow X^{\bullet}$ is a morphism in $\mathrm{K}(\mathscr{A})$. Two such roofs $X^{\bullet} \stackrel{f^{\bullet}}{\longleftarrow} Z^{\bullet} \stackrel{g^{\bullet}}{\longrightarrow} Y^{\bullet}$ and $X^{\prime \bullet} \stackrel{f^{\bullet}}{\longleftarrow} Z^{\prime \bullet} \stackrel{g^{\bullet}}{\longrightarrow} Y^{\bullet \bullet}$ are equivalent if there exists a commutative diagram

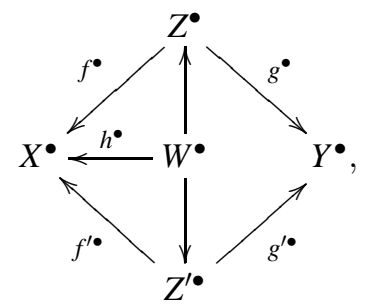

where $h^{\bullet}$ is an $F$-quasi-isomorphism. Note that the diagram of the form $X^{\bullet} \stackrel{f^{\bullet}}{\longleftarrow} Z^{\bullet} \stackrel{g^{\bullet}}{\longrightarrow} Y^{\bullet}$ will be called a left roof.

\section{Homological properties of relative derived categories}

In this section, we give some basic properties of relative derived categories.

The following lemma is well-known.

Lemma 3.1. [17, Lemma 2.4] Let $\mathcal{T}_{1}$ and $\mathcal{T}_{2}$ be triangulated subcategories of a triangulated category $\mathcal{T}$, and $\mathcal{T}_{2}$ a full triangulated subcategory of $\mathcal{T}_{1}$. Then there is an isomorphism of triangulated categories $\left(\mathcal{T} / \mathcal{T}_{2}\right) /\left(\mathcal{T}_{1} / \mathcal{T}_{2}\right) \simeq \mathcal{T} / \mathcal{T}_{1}$

Set $\mathscr{N}^{\prime}=\left\{X^{\bullet} \mid X^{\bullet}\right.$ is an acyclic complex $\}$. Then $\mathscr{N}^{\prime}$ is also a null system. Consequently, $\mathscr{N}$ is a triangulated subcategory of $\mathscr{N}^{\prime}$. By Lemma 3.1, it follows that

$$
\mathrm{D}^{\mathrm{b}}(\mathscr{A})=\mathrm{K}(\mathscr{A}) / \mathscr{N}^{\prime} \simeq(\mathrm{K}(\mathscr{A}) / \mathscr{N}) /\left(\mathscr{N}^{\prime} / \mathscr{N}\right) \simeq \mathrm{D}_{\mathrm{F}}^{\mathrm{b}}(\mathscr{A}) /\left(\mathscr{N}^{\prime} / \mathscr{N}\right)
$$

where $\mathrm{D}^{\mathrm{b}}(\mathscr{A})$ is the derived category of $\mathscr{A}$. Then the usual derived category is a quotient category of relative derived category.

As is known, if $F$ has enough projective objects, then an exact sequence $0 \rightarrow A \rightarrow B \rightarrow C \rightarrow 0$ is $F$-exact if and only if $0 \rightarrow \operatorname{Hom}(P, A) \rightarrow \operatorname{Hom}(P, B) \rightarrow \operatorname{Hom}(P, C) \rightarrow 0$ is exact for all $P$ in $\mathcal{P}(F)$. We have the following lemma.

Lemma 3.2. If $F$ has enough projective objects, then a complex $X^{\bullet}$ is an $F$-acyclic complex if and only if $\operatorname{Hom}\left(P, X^{\bullet}\right)$ is an acyclic complex for all $P$ in $\mathcal{P}(F)$.

It is well known that the categories $\mathrm{D}^{-}(\mathscr{A}), \mathrm{D}^{+}(\mathscr{A})$ and $\mathrm{D}^{\mathrm{b}}(\mathscr{A})$ are full subcategories of $\mathrm{D}(\mathscr{A})$. This remains true for $\mathrm{D}_{\mathrm{F}}^{*}(\mathscr{A})$ in $\mathrm{D}_{\mathrm{F}}(\mathscr{A})$, where $\star=+,-, b$. We will prove these from a series of observations to realize these facts. 
Proposition 3.3. 47, Lemma 10.1.13] Let $\mathcal{S}$ be a multiplicative system of $\mathcal{C}$ and $\mathcal{D}$ a full subcategory of $C$. Assume that $\mathcal{S} \cap \mathcal{D}$ is a multiplicative system of $\mathcal{D}$, and one of the following conditions is satisfied:

(1) If $s: X^{\prime} \rightarrow X$ is a morphism in $\mathcal{S}$, with $X \in \mathcal{D}$, then there is a morphism $f: X^{\prime \prime} \rightarrow X^{\prime}$ in $\mathcal{C}$ such that $X^{\prime \prime} \in \mathcal{D}$ and $f s \in \mathcal{S}$.

(2) If $s: X \rightarrow X^{\prime}$ is a morphism in $\mathcal{S}$ with $X \in \mathcal{D}$, then there is a morphism $f: X^{\prime} \rightarrow X^{\prime \prime}$ in $C$ such that $X^{\prime \prime} \in \mathcal{D}$ and $s f \in \mathcal{S}$.

Then the natural functor $(\mathcal{S} \cap \mathcal{D})^{-1} \mathcal{D} \rightarrow \mathcal{S}^{-1} \mathrm{C}$ is fully faithful. That is, $(\mathcal{S} \cap \mathcal{D})^{-1} \mathcal{D}$ is considered as a full subcategory of $\mathcal{S}^{-1} \mathrm{C}$.

Corollary 3.4. (1) If $X^{\bullet \bullet} \rightarrow X^{\bullet}$ is an $F$-quasi-isomorphism, $X^{\bullet} \in \mathrm{K}^{\mathrm{b}}(\mathscr{A}), X^{\bullet \bullet} \in \mathrm{K}^{+}(\mathscr{A})$, then there is an $F$-quasi-isomorphism $X^{\prime \prime} \bullet X^{\prime \bullet}$ such that $X^{\prime \prime} \bullet \in \mathrm{K}^{\mathrm{b}}(\mathscr{A})$.

(2) If $Y^{\bullet} \rightarrow X^{\bullet}$ is an F-quasi-isomorphism, $X^{\bullet} \in \mathrm{K}^{-}(\mathscr{A}), Y^{\bullet} \in \mathrm{K}(\mathscr{A})$, then there is an F-quasiisomorphism $Y^{\prime \bullet} \rightarrow Y^{\bullet}$ such that $Y^{\prime \bullet} \in \mathrm{K}^{-}(\mathscr{A})$.

Proof. The proof of (1) is similar to the one of (2). We only need to prove the second case.

(2) Let $X^{\bullet}$ be in $\mathrm{K}^{-}(\mathscr{A})$, we may assume that $X^{i}=0$ for $i>0$. Then $X^{\bullet}$ has the form

$$
\cdots \rightarrow X^{-1} \rightarrow X^{0} \rightarrow 0 \rightarrow \cdots .
$$

Let $f^{\bullet}: X^{\bullet} \rightarrow Y^{\bullet}$ be a map of complexes. Then we have the following commutative diagram

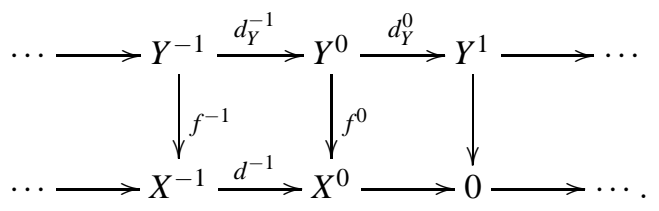

Since $f^{\bullet}$ is an $F$-quasi-isomorphism, it follows that $M\left(f^{\bullet}\right)$ is an $F$-acyclic complex.

$$
M\left(f^{\bullet}\right): \cdots \rightarrow X^{-1} \oplus Y^{0} \rightarrow X^{0} \oplus Y^{1} \rightarrow Y^{2} \rightarrow \cdots
$$

Decompose $M\left(f^{\bullet}\right)$ into the direct sum of two $F$-exact complexes:

$$
\cdots \rightarrow X^{-1} \oplus Y^{0} \rightarrow X^{0} \oplus Y^{1} \rightarrow \operatorname{Imd}_{Y}^{1} \rightarrow 0 \rightarrow \cdots
$$

and

$$
\cdots \rightarrow 0 \rightarrow \operatorname{Imd}_{Y}^{2} \rightarrow Y^{3} \rightarrow \cdots .
$$

Then we get the following commutative diagram:

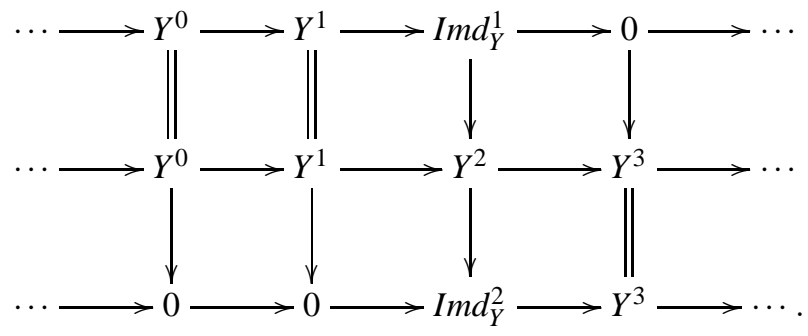

Set

$$
Y^{\prime \bullet}: \cdots \rightarrow Y^{0} \rightarrow Y^{1} \rightarrow \operatorname{Imd}^{1} \rightarrow 0 \rightarrow \cdots .
$$

Then $Y^{\prime \bullet}$ and $Y^{\bullet}$ are $F$-quasi-isomorphic with $Y^{\prime \bullet} \in \mathrm{K}^{-}(\mathscr{A})$.

Proposition 3.5. $\mathrm{D}_{\mathrm{F}}^{\mathrm{b}}(\mathscr{A}), \mathrm{D}_{\mathrm{F}}^{-}(\mathscr{A})$ and $\mathrm{D}_{\mathrm{F}}{ }^{+}(\mathscr{A})$ are full subcategories of $\mathrm{D}_{\mathrm{F}}(\mathscr{A})$.

Proof. By definition, $\mathrm{D}_{\mathrm{F}}^{-}(\mathscr{A})=\mathrm{K}^{-}(\mathscr{A}) /\left(\mathscr{N} \cap \mathrm{K}^{-}(\mathscr{A})\right)$, since $\mathrm{K}^{-}(\mathscr{A})$ is a full subcategory of $\mathrm{K}(\mathscr{A})$. Moreover, if $Y^{\bullet} \rightarrow X^{\bullet}$ is an $F$-quasi-isomorphism, $X^{\bullet} \in \mathrm{K}^{-}(\mathscr{A}), Y^{\bullet} \in \mathrm{K}(\mathscr{A})$, then there is an $F$-quasi-isomorphism $Y^{\prime \bullet} \rightarrow X^{\bullet}$ by Corollary 3.4(2). From Proposition 3.3, we deduce that $\mathrm{D}_{\mathrm{F}}{ }^{-}(\mathscr{A})$ is a full subcategory of $\mathrm{D}_{\mathrm{F}}(\mathscr{A})$. In a similar way, $\mathrm{D}_{\mathrm{F}}{ }^{-}(\mathscr{A})$ and $\mathrm{D}_{\mathrm{F}}{ }^{+}(\mathscr{A})$ are full subcategories of $\mathrm{D}_{\mathrm{F}}(\mathscr{A})$.

The following results make the morphisms in $\mathrm{D}_{\mathrm{F}}(\mathscr{A})$ to the morphisms in $\mathrm{K}(\mathscr{A})$ to understand. 
Lemma 3.6. Let $P^{\bullet} \in \mathrm{K}^{-}(\mathcal{P}(F))$ and $f^{\bullet}: X^{\bullet} \rightarrow P^{\bullet}$ an $F$-quasi-isomorphism. Then there is a map $g^{\bullet}: P^{\bullet} \rightarrow X^{\bullet}$ such that $g^{\bullet} f^{\bullet}$ is homotopic to id $P^{\bullet}$.

Proof. Let $f^{\bullet}: X^{\bullet} \rightarrow P^{\bullet}$ be an $F$-quasi-isomorphism. Then we have a distinguished triangle $X^{\bullet} \stackrel{f^{\bullet}}{\rightarrow} P^{\bullet} \stackrel{h^{\bullet}}{\rightarrow} M\left(f^{\bullet}\right) \stackrel{l^{\bullet}}{\rightarrow} X^{\bullet}[1]$ in $\mathrm{K}^{-}(\mathcal{P}(F))$, where the mapping cone $M\left(f^{\bullet}\right):=X^{\bullet}[1] \oplus P^{\bullet}$ is an $F$-acyclic complex. For $P^{\bullet} \in \mathrm{K}^{-}(\mathcal{P}(F))$, without loss of generality we can assume that $P^{i}=0$ for $i>n$. Therefore, we have the following commutative diagram

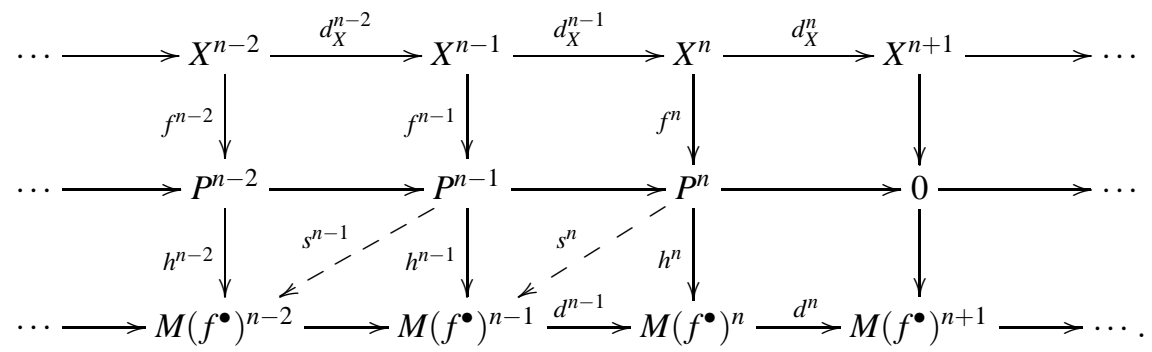

From the above diagram, we have $h^{n} d_{M\left(f^{\bullet}\right)}^{n}=0$. By the (relative) Comparision Theorem, it follows that $h^{\bullet}$ is a null-homotopic. Then there is a chain map $s^{\bullet}=\left(g^{\bullet}, v^{\bullet}\right): P^{\bullet} \rightarrow X^{\bullet} \oplus P^{\bullet}[1]$ such that $h^{\bullet}=s^{\bullet} d_{M\left(f^{\bullet}\right)}+$ $d_{P}^{\bullet} s^{\bullet}$, where $d_{M\left(f^{\bullet}\right)}^{i}=\left(\begin{array}{cc}-d_{X}^{i+1} & f^{i+1} \\ 0 & d_{P}^{i}\end{array}\right)$. Thus the above equation yields the following equations

$$
-g d_{X}+d_{P} g=0, g f+v d_{P}+d_{P} v=i d .
$$

It follows that $g$ is a chain map and $i d=g^{\bullet} f^{\bullet}$ in $\mathrm{K}^{-}(\mathscr{A})$.

Proposition 3.7. Let $P^{\bullet} \in \mathrm{K}^{-}(\mathcal{P}(F))$ and $X^{\bullet} \in \mathrm{K}(\mathscr{A})$. Then

$$
\operatorname{Hom}_{\mathrm{K}(\mathscr{A})}\left(P^{\bullet}, X^{\bullet}\right) \simeq \operatorname{Hom}_{\mathrm{D}_{\mathrm{F}}(\mathscr{A})}\left(P^{\bullet}, X^{\bullet}\right) .
$$

Proof. Define a map $\varphi: \operatorname{Hom}_{\mathrm{K}(\mathscr{A})}\left(P^{\bullet}, X^{\bullet}\right) \rightarrow \operatorname{Hom}_{\mathrm{D}_{\mathrm{F}}(\mathscr{A})}\left(P^{\bullet}, X^{\bullet}\right)$ which sends $f^{\bullet}$ to $f^{\bullet} /$ id. If $f^{\bullet}: P^{\bullet} \rightarrow X^{\bullet}$ satisfies $f^{\bullet} /$ id $=0$, then there is an $F$-quasi-isomorphism $t^{\bullet}: X^{\boldsymbol{\prime}^{\bullet}} \rightarrow P^{\bullet}$ such that $t^{\bullet} f^{\bullet}$ is homotopic to zero. By Lemma 3.6, there is an $F$-quasi-isomorphism $g^{\bullet}: P^{\bullet} \rightarrow X^{\boldsymbol{\bullet}^{\bullet}}$ such that $g^{\bullet} t^{\bullet}$ is homotopic to $i d_{P^{\bullet}}$. Thus, we get $g^{\bullet} t^{\bullet} f^{\bullet}=0$. Consequently, $f^{\bullet}=0$ in $\mathrm{K}(\mathscr{A})$. This implies that $\varphi$ is an injective map. For any $f^{\bullet} / s^{\bullet}$ is in $\operatorname{Hom}_{\mathrm{D}_{\mathrm{F}}(\mathscr{A})}\left(P^{\bullet}, X^{\bullet}\right)$, by Lemma 3.6, there is an $F$-quasi-isomorphism $g^{\bullet}$ such that $g^{\bullet} s^{\bullet}$ is homotopic to $i d_{P^{\bullet}}$. Consequently, $f^{\bullet} / s^{\bullet}=f^{\bullet} g^{\bullet} / i d_{P^{\bullet}}$. Therefore, $\varphi$ is surjective.

Suppose that the functor $F$ has enough projective objects. For any $X$ in $\mathscr{A}$, take a minimal $F$-projective resolution $P_{X}$ of $X$, that is, there exists an $F$-exact sequence

$$
\cdots \rightarrow P^{-2} \rightarrow P^{-1} \rightarrow P^{0} \rightarrow X \rightarrow 0,
$$

with $P^{i} F$-projectives for $i>0$. Then we define $\operatorname{Ext}_{F}^{i}(X, Y)$ to be the $i$-th homology of the complex

$$
0 \rightarrow \operatorname{Hom}\left(P^{0}, Y\right) \rightarrow \operatorname{Hom}\left(P^{-1}, Y\right) \rightarrow \cdots \rightarrow \operatorname{Hom}\left(P^{-i}, Y\right) \rightarrow \cdots .
$$

Lemma 3.8. Suppose that $F$ has enough projective objects. Let $X$ and $Y$ be in $\mathscr{A}$. Then

$$
\operatorname{Ext}_{F}^{i}(X, Y) \simeq \operatorname{Hom}_{\mathrm{D}_{\mathrm{F}}^{\mathrm{b}}(\mathscr{A})}(X, Y[i])
$$

for all $i \in \mathbb{Z}$.

Proof. Take a minimal $F$-projective resolution $P_{X}^{\bullet}$ of $X$ with $P_{X}^{\bullet} \in \mathrm{K}^{-, b}(\mathscr{A})$. Then we have the following

$$
\begin{array}{r}
\operatorname{Hom}_{\mathrm{D}_{\mathrm{F}}^{\mathrm{b}}(\mathscr{A})}(X, Y[i]) \simeq \operatorname{Hom}_{\mathrm{D}_{\mathrm{F}}^{\mathrm{b}}(\mathscr{A})}\left(P_{X}^{\bullet}, Y[i]\right) \simeq \operatorname{Hom}_{\mathrm{K}(\mathscr{A})}\left(P_{X}^{\bullet}, Y[i]\right) \\
\simeq H^{i}\left(\operatorname{Hom}\left(P_{X}, Y\right)\right)=\operatorname{Ext}_{F}^{i}(X, Y) .
\end{array}
$$


Define $\mathrm{K}^{-, F b}(\mathcal{P}(F))$ to be the subcategory of $\mathrm{K}^{-, b}(\mathcal{P}(F))$ by

$\mathrm{K}^{-, F b}(\mathcal{P}(F))=\left\{X^{\bullet} \in \mathrm{K}^{-}(\mathcal{P}(F)) \mid \exists n \in \mathbb{Z}\right.$ such that $H^{i}\left(\operatorname{Hom}_{A}\left(P, X^{\bullet}\right)\right)=0$ for $\left.i \leq n, \forall P \in \mathcal{P}(F)\right\}$.

It is easy to easy that for $X^{\bullet} \in \mathrm{K}^{-}(\mathcal{P}(F))$ and $\forall i \in \mathbb{Z}$, if $H^{i}\left(\operatorname{Hom}_{A}\left(P, X^{\bullet}\right)\right)=0$, for $i \leq n, \forall P \in \mathcal{P}(F)$, then $H^{i}\left(X^{\bullet}\right)=0$.

Proposition 3.9. Suppose $F$ has enough projective objects. Then the natural functor induces two triangle equivalences:

$$
\mathrm{D}_{\mathrm{F}}{ }^{-}(\mathscr{A}) \simeq \mathrm{K}^{-}(\mathcal{P}(F)), \mathrm{D}_{\mathrm{F}}^{\mathrm{b}}(\mathscr{A}) \simeq \mathrm{K}^{-, F b}(\mathcal{P}(F)) .
$$

Proof. For more details, we refer the reader to [47, Theorem 10.4.8] and [34, Proposition 6.3.1].

Let $H: \mathscr{A} \rightarrow \mathrm{D}_{\mathrm{F}}^{\mathrm{b}}(\mathscr{A})$ be the composition of the embedding $\mathscr{A} \rightarrow \mathrm{K}^{\mathrm{b}}(\mathscr{A})$ with the localization functor $\mathrm{K}^{\mathrm{b}}(\mathscr{A}) \rightarrow \mathrm{D}_{\mathrm{F}}^{\mathrm{b}}(\mathscr{A})$. Then we have the following proposition.

Lemma 3.10. The functor $H: \mathscr{A} \rightarrow \mathrm{D}_{\mathrm{F}}^{\mathrm{b}}(\mathscr{A})$ is fully faithful.

Proof. It suffices to show that $H(X, Y): \operatorname{Hom}_{\mathscr{A}}(X, Y) \simeq \operatorname{Hom}_{\mathrm{D}_{\mathrm{F}}^{\mathrm{b}}(\mathscr{A})}(X, Y)$ as abelian groups. Let $u \in \operatorname{Hom}_{\mathscr{A}}(X, Y)$ such that $H(u)=0$. Then we have the following diagram

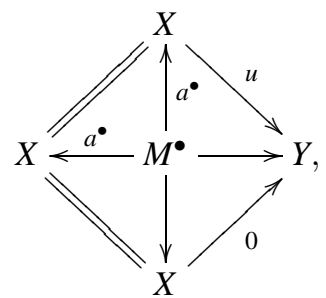

where $a^{\bullet}: M^{\bullet} \rightarrow X$ is an $F$-quasi-isomorphism such that $a^{\bullet} u=0$. Therefore, $H^{0}(a): H^{0}\left(M^{\bullet}\right) \rightarrow X$ is an isomorphism and $H^{0}(a) u=0$. This implies that $u=0$ and $H$ is a faithful functor. We take a left roof $X \stackrel{s^{\bullet}}{\longleftarrow} U^{\bullet} \stackrel{f^{\bullet}}{\longrightarrow} Y$ in $\operatorname{Hom}_{\mathrm{D}_{\mathrm{F}}^{\mathrm{b}}(\mathscr{A})}(X, Y)$, where $U^{\bullet} \in \mathrm{K}^{\mathrm{b}}(\mathscr{A})$ and $s^{\bullet}$ is an $F$-quasi-isomorphism. Consequently, $H^{0}\left(s^{\bullet}\right): H^{0}\left(U^{\bullet}\right) \rightarrow X$ is an isomorphism in $\mathscr{A}$. Set $g=H^{0}\left(s^{\bullet}\right)^{-1} H^{0}\left(f^{\bullet}\right): X \rightarrow Y$. Consider the truncation complex $W^{\bullet}: \cdots \rightarrow U^{-2} \rightarrow U^{-1} \rightarrow \operatorname{Kerd}^{0} \rightarrow 0$ of $U^{\bullet}$ and the canonical chain map $i^{\bullet}: W^{\bullet} \rightarrow U^{\bullet}$. It is easy to see that $i^{\bullet}$ is an $F$-quasi-isomorphism. Since $s^{\bullet}$ is an $F$-quasi-isomorphism, it follows that $i^{\bullet} s^{\bullet}$ is an $F$-quasi-isomorphism. To get the following commutative diagram

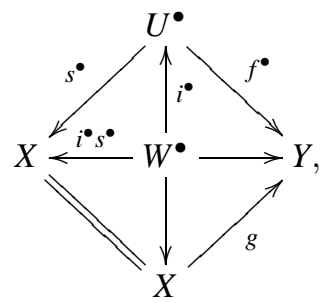

it is sufficient to show that $i^{\bullet} f^{\bullet}=i^{\bullet} s^{\bullet} g$. From the following commutative diagram

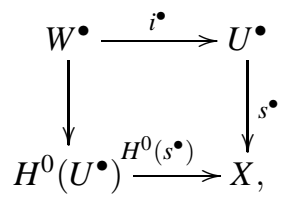

we conclude that $i^{\bullet} \boldsymbol{s}^{\bullet} g=i^{\bullet} s^{\bullet} H^{0}\left(s^{\bullet}\right)^{-1} H^{0}\left(f^{\bullet}\right)=i^{\bullet} f^{\bullet}$. Then $L(g / i d)=f^{\bullet} / s^{\bullet}$ and $L$ is a full functor. 


\section{Triangles in relative derived categories}

In this section, we describe the triangulated structure of $\mathrm{D}_{\mathrm{F}}^{\mathrm{b}}(\mathscr{A})$, where $F$ has enough projectives.

We have the following proposition.

Proposition 4.1. Suppose that

$$
0 \rightarrow X^{\bullet} \stackrel{f^{\bullet}}{\rightarrow} Y^{\bullet} \stackrel{g^{\bullet}}{\rightarrow} Z^{\bullet} \rightarrow 0
$$

is an $F$-exact sequence of complexes in $\mathrm{C}(\mathscr{A})$, that is,

$$
0 \rightarrow X^{i} \rightarrow Y^{i} \rightarrow Z^{i} \rightarrow 0
$$

is an $F$-exact sequence for each $i$. Let $M\left(f^{\bullet}\right)$ be the mapping cone of $f^{\bullet}$, and let $\phi^{i}: M\left(f^{\bullet}\right)^{i}=X^{i+1} \oplus Y^{i} \rightarrow$ $Z^{i}$ be the morphism $\left(\begin{array}{c}0 \\ g^{i}\end{array}\right)$. Then $\phi^{\bullet}: M\left(f^{\bullet}\right) \rightarrow Z^{\bullet}$ is a morphism of complexes, $\alpha\left(f^{\bullet}\right) \phi^{\bullet}=g^{\bullet}$, and $\phi^{\bullet}$ is an F-quasi-isomorphism. Moreover,

$$
X^{\bullet} \rightarrow Y^{\bullet} \rightarrow Z^{\bullet} \rightarrow X^{\bullet}[1]
$$

is a distinguished triangle in $\mathrm{D}_{\mathrm{F}}^{\mathrm{b}}(\mathscr{A})$.

Proof. It is straightforward to see that $\phi^{\bullet}$ is a morphism of complexes. We know that there is a diagram of maps of complexes:

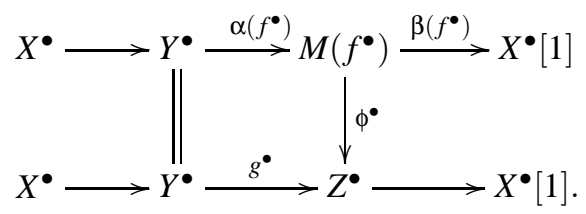

It suffices to show that $M\left(f^{\bullet}\right) \stackrel{\phi^{\bullet}}{\rightarrow} Z^{\bullet}$ is an $F$-quasi-isomorphism. Moreover, there is an $F$-exact sequence of complexes $0 \rightarrow M\left(i d_{X} \bullet \stackrel{\rho^{\bullet}}{\rightarrow} M\left(f^{\bullet}\right) \stackrel{\phi^{\bullet}}{\rightarrow} Z^{\bullet} \rightarrow 0\right.$, where $\rho^{i}=\left(\begin{array}{cc}i d & 0 \\ 0 & f^{i}\end{array}\right)$. By assumption, for each $P \in \mathcal{P}(F)$, it follows that

$$
0 \rightarrow\left(P, M\left(i d_{X^{\bullet}}\right)\right) \rightarrow\left(P, M\left(f^{\bullet}\right)\right) \rightarrow\left(P, Z^{\bullet}\right) \rightarrow 0
$$

is an exact sequence of complexes. Since $H^{i}\left(\left(P, M\left(i d_{X} \bullet\right)\right)\right)=0$, we deduce that $\phi^{\bullet}$ is an $F$-quasiisomorphism. Therefore, $\phi^{\bullet}$ is an isomorphism in $\mathrm{D}_{\mathrm{F}}^{\mathrm{b}}(\mathscr{A})$. Therefore,

$$
X^{\bullet} \stackrel{f^{\bullet}}{\rightarrow} Y^{\bullet} \stackrel{g^{\bullet}}{\rightarrow} Z^{\bullet} \stackrel{h^{\bullet}}{\rightarrow} X^{\bullet}[1]
$$

is a distinguished triangle in $\mathrm{D}_{\mathrm{F}}^{\mathrm{b}}(\mathscr{A})$, where $h^{\bullet}=\phi^{\bullet-1} \beta\left(f^{\bullet}\right)$.

\section{Relative stable categories and relative derived categories}

In this section, we assume that $F$ has enough projectives and injectives and $\mathcal{P}(F)=I(F)$. Then $\mathscr{A}$ is a Frobenius category. Let $\mathscr{A} / \mathscr{P}(F)$ denote the stable category of $\mathscr{A}$. By [21, Theorem 2.6], $\mathscr{A} / \mathscr{P}(F)$ is a triangulated category. The shift functor of $\mathscr{A} / \mathcal{P}(F)$ is $\Omega_{F}^{-1}$, where $\Omega_{F}$ is the $F$-syzygy functor. Since $\mathcal{P}(F)$ is closed under taking direct summands, it follows that $\mathrm{K}^{\mathrm{b}}(\mathcal{P}(F))$ is a thick subcategory of $\mathrm{D}_{\mathrm{F}}^{\mathrm{b}}(\mathscr{A})$.

We are now ready to state our main result of this section.

Theorem 5.1. The quotient category

$$
\mathrm{D}_{\mathrm{F}}^{\mathrm{b}}(\mathscr{A}) / \mathrm{K}^{\mathrm{b}}(\mathscr{P}(F))
$$

is equivalent as a triangulated category to $\mathscr{A} / \mathcal{P}(F)$. 
Proof. The proof is similar to that of [41, Theorem 2.1]. For convenience, we give more details.

Consider the additive functor

$$
L^{\prime}: \mathscr{A} \rightarrow \mathrm{D}_{\mathrm{F}}^{\mathrm{b}}(\mathscr{A}) / \mathrm{K}^{\mathrm{b}}(\mathcal{P}(F))
$$

obtained by the composition of the embedding $\mathscr{A} \rightarrow \mathrm{D}_{\mathrm{F}}^{\mathrm{b}}(\mathscr{A})$ with the localization functor $\mathrm{D}_{\mathrm{F}}^{\mathrm{b}}(\mathscr{A}) \rightarrow$ $\mathrm{D}_{\mathrm{F}}^{\mathrm{b}}(\mathscr{A}) / \mathrm{K}^{\mathrm{b}}(\mathscr{P}(F))$. The natural inclusion of $\mathscr{A}$ sends $F$-projective objects to zero in the quotient category, hence the functor $L$ factors through the stable category, inducing an additive functor $L: \mathscr{A} / \mathcal{P}(F) \simeq$ $\mathrm{D}_{\mathrm{F}}^{\mathrm{b}}(\mathscr{A}) / \mathrm{K}^{\mathrm{b}}(\mathcal{P}(F))$.

The proof will be divided into three steps.

Step1. $L$ is an exact functor. Consider a distinguished triangle

$$
X \rightarrow Y \rightarrow Z \rightarrow \Omega_{F}^{-1}(X)
$$

in $\mathscr{A} / \mathscr{P}(F)$. This distinguished triangle is from the following pushout diagram:

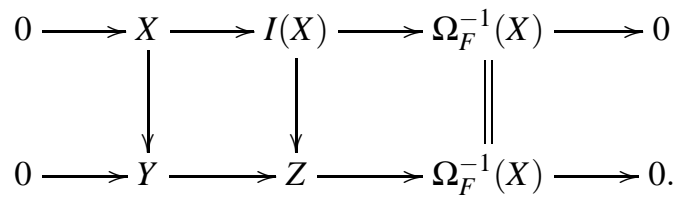

Since $F$-exact sequences are closed under pushout, it follows that $0 \rightarrow Y \rightarrow Z \rightarrow \Omega_{F}^{-1}(X) \rightarrow 0$ is an $F$ exact sequence. From $F$-exact sequences $0 \rightarrow X \rightarrow I(X) \rightarrow \Omega_{F}^{-1}(X) \rightarrow 0$ and $0 \rightarrow I(X) \rightarrow Z \rightarrow \Omega_{F}^{-1}(X) \rightarrow$ 0 , we have distinguished triangles $X \rightarrow I(X) \rightarrow \Omega_{F}^{-1}(X) \rightarrow X[1]$ and $Y \rightarrow Z \rightarrow \Omega_{F}^{-1}(X) \rightarrow Y[1]$ in $\mathrm{D}_{\mathrm{F}}^{\mathrm{b}}(\mathscr{A})$.

Since $L^{\prime}(I(X))=0$ in $\mathrm{D}_{\mathrm{F}}^{\mathrm{b}}(\mathscr{A}) / \mathrm{K}^{\mathrm{b}}(\mathscr{P}(F))$, we deduce that

$$
L(X) \rightarrow L(Y) \rightarrow L(Z) \rightarrow L(X)[1]
$$

is a distinguished triangle in $\mathrm{D}_{\mathrm{F}}^{\mathrm{b}}(\mathscr{A}) / \mathrm{K}^{\mathrm{b}}(\mathscr{P}(F))$. Consequently, $L$ is an exact functor.

Step2. $L$ is a fully faithful functor. Firstly, $L^{\prime}$ is a full functor. We see that the map

$$
\operatorname{Hom}_{\mathscr{A}}(X, Y) \rightarrow \operatorname{Hom}_{\mathrm{D}_{\mathrm{F}}^{\mathrm{b}}(\mathscr{A}) / \mathrm{K}^{\mathrm{b}}(\mathscr{P}(F))}(X, Y)
$$

sending $f: X \rightarrow Y$ to $f /$ id is surjective, where $f / i d$ is the left roof

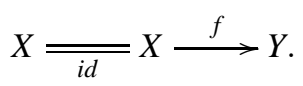

Take a left roof $X \stackrel{s}{\longleftarrow} Z^{\bullet} \stackrel{g}{\longrightarrow} Y$ in $\operatorname{Hom}_{\mathrm{D}_{\mathrm{F}}^{\mathrm{b}}(\mathscr{A}) / \mathrm{K}^{\mathrm{b}}(\mathscr{P}(F))}(X, Y)$, where $Z^{\bullet} \in \mathrm{D}_{\mathrm{F}}^{\mathrm{b}}(\mathscr{A})$. It follows that there is a distinguished triangle

$$
Z^{\bullet} \stackrel{s}{\rightarrow} X \rightarrow M(s) \rightarrow Z^{\bullet}[1]
$$

in $\mathrm{D}_{\mathrm{F}}^{\mathrm{b}}(\mathscr{A})$ such that the mapping cone $M(s)$ is in $\mathrm{K}^{\mathrm{b}}(\mathscr{P}(F)$ ). Applying the cohomological functor $\operatorname{Hom}_{\mathrm{D}_{\mathrm{F}}^{\mathrm{b}}(\mathscr{A}) / \mathrm{K}^{\mathrm{b}}(\mathscr{P}(F))}(-, Y)$ to the distinguished triangle $Z^{\bullet} \stackrel{s}{\rightarrow} X \rightarrow M(s) \rightarrow Z^{\bullet}[1]$, we have an exact sequence

$$
\begin{array}{r}
\cdots \rightarrow \operatorname{Hom}_{\mathrm{D}_{\mathrm{F}}^{\mathrm{b}}(\mathscr{A}) / \mathrm{K}^{\mathrm{b}}(\mathscr{P}(F))}(M(s), Y) \rightarrow \operatorname{Hom}_{\mathrm{D}_{\mathrm{F}}^{\mathrm{b}}(\mathscr{A}) / \mathrm{K}^{\mathrm{b}}(\mathcal{P}(F))}(X, Y) \\
\stackrel{(s, Y)}{\rightarrow} \operatorname{Hom}_{\mathrm{D}_{\mathrm{F}}^{\mathrm{b}}(\mathscr{A}) / \mathrm{K}^{\mathrm{b}}(\mathscr{P}(F))}\left(Z^{\bullet}, Y\right) \rightarrow \operatorname{Hom}_{\mathrm{D}_{\mathrm{F}}^{\mathrm{b}}(\mathscr{A}) / \mathrm{K}^{\mathrm{b}}(\mathscr{P}(F))}(M(s)[-1], Y) \rightarrow \cdots
\end{array}
$$

Furthermore,

$$
\operatorname{Hom}_{\mathrm{D}_{\mathrm{F}}^{\mathrm{b}}(\mathscr{A}) / \mathrm{K}^{\mathrm{b}}(\mathcal{P}(F))}(M(s)[-1], Y)=0 \quad \text { since } M(s)[-1] \quad \text { is in } \mathrm{K}^{\mathrm{b}}(\mathcal{P}(F)) .
$$


Then we get $g=s f$ and the following commutative diagram

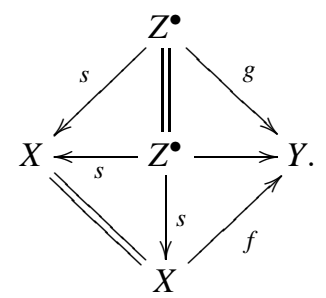

Therefore, $f / i d$ and $g / s$ are equivalent in $\mathrm{D}_{\mathrm{F}}^{\mathrm{b}}(\mathscr{A}) / \mathrm{K}^{\mathrm{b}}(\mathcal{P}(F))$. This implies that $L$ is a fully functor.

We claim that if $X$ is an object of $\mathscr{A}$, then $L(X)=0$ if and only if $X=0$. Indeed, no non-projective object is isomorphic to an object of $\mathrm{K}^{\mathrm{b}}(\mathscr{P}(F))$.

Assume that $\alpha: X \rightarrow Y$ is in $\mathscr{A} / \mathcal{P}(F)$ such that $L(\alpha)=0$. There is a distinguished triangle $X \stackrel{\alpha}{\rightarrow} Y \stackrel{x}{\rightarrow}$ $Z \rightarrow \Omega_{F}^{-1}(X)$ in $\mathscr{A} / \mathcal{P}(F)$. Since $L$ is an exact functor, we see that $L(X) \stackrel{L(\alpha)}{\rightarrow} L(Y) \rightarrow L(Z) \rightarrow L(X)[1]$ is a distinguished triangle in $\mathrm{D}_{\mathrm{F}}^{\mathrm{b}}(\mathscr{A}) / \mathrm{K}^{\mathrm{b}}(\mathcal{P}(F))$. Since $L(\alpha)=0$, we conclude that $L(Z) \simeq(L(Y) \oplus L(X)[1])$. Thus, we have the following commutative diagram

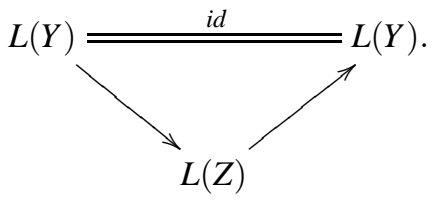

Since $L$ is full, we get the following diagram

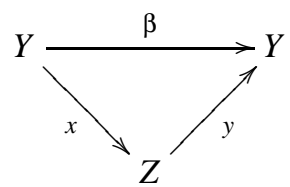

such that $L(\beta)=i d=L(x y)$. Then we have a distinguished triangle $Y \stackrel{x y}{\rightarrow} Y \rightarrow V \rightarrow \Omega_{F}^{-1}(Y)$ in $\mathscr{A} / \mathcal{P}(F)$. Thus, we get a distinguished triangle $L(Y) \stackrel{i d}{\rightarrow} L(Y) \rightarrow L(V) \rightarrow L(Y)[1]$ in $\mathrm{D}_{\mathrm{F}}^{\mathrm{b}}(\mathscr{A}) / \mathrm{K}^{\mathrm{b}}(\mathcal{P}(F))$ Consequently, $L(V)=0$. Therefore, we have $V=0$ by our claim. It follows that $x y$ is an isomorphism and $x$ is split monomorphism. Then $\alpha=0$ and $L$ is a faithful functor.

Step3. $L$ is dense. Let $X^{\bullet}$ be in $\mathrm{D}_{\mathrm{F}}^{\mathrm{b}}(\mathscr{A}) / \mathrm{K}^{\mathrm{b}}(\mathscr{P}(F))$. Then $X^{\bullet}$ is in $\mathrm{D}_{\mathrm{F}}^{\mathrm{b}}(\mathscr{A})$. Take an $F$-projective resolution $P_{X}^{\bullet}$ of $X^{\bullet}$ with $P_{X}^{\bullet} \in \mathrm{K}^{-, F b}(\mathcal{P}(F))$. Without loss of generality, we assume that $P_{X}^{\bullet}$ has the following form:

$$
\cdots \rightarrow P_{X}^{-r-2} \rightarrow P_{X}^{-r-1} \rightarrow P_{X}^{-r} \stackrel{d^{-r}}{\rightarrow} \cdots \rightarrow P_{X}^{0} \rightarrow 0 \rightarrow \cdots
$$

such that $H^{i}\left(Q, P_{X}^{\bullet}\right)=0$ for $i<-r$ with any $Q \in \mathcal{P}(F)$. Then we deduce that

$$
\cdots \rightarrow P_{X}^{-r-2} \rightarrow \operatorname{Im}\left(d_{X}^{-r-2}\right) \rightarrow 0 \rightarrow \cdots
$$

is an $F$-acyclic complex. Therefore, the complex $P_{X}^{\bullet}$ is isomorphic in $\mathrm{D}_{\mathrm{F}}^{\mathrm{b}}(\mathscr{A})$ to a complex of the form

$$
0 \rightarrow \operatorname{Im}\left(d_{X}^{-r-2}\right) \rightarrow P_{X}^{-r-1} \stackrel{d^{-r-1}}{\rightarrow} P_{X}^{-r} \rightarrow \cdots \rightarrow P_{X}^{0} \rightarrow 0 \rightarrow \cdots .
$$

From the following commutative diagram

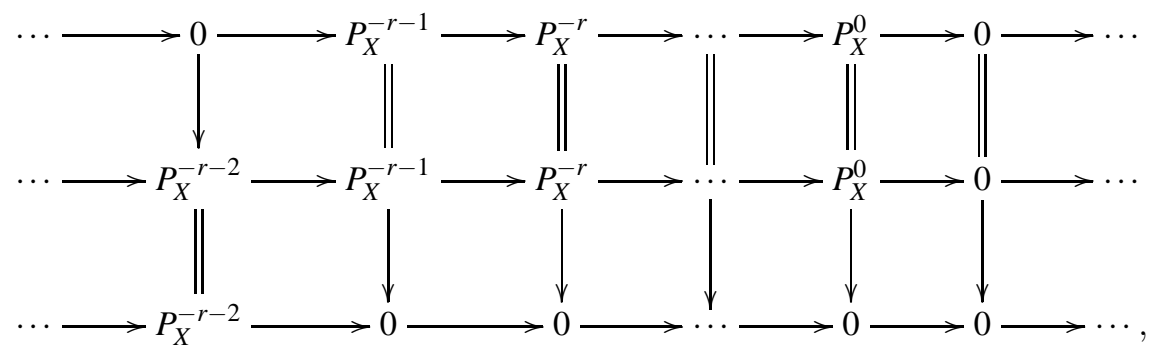


we conclude that $\operatorname{Im}\left(d_{X}^{-r-2}\right)[r+2] \simeq P_{X}^{\bullet} \simeq X^{\bullet}$ in $\mathrm{D}_{\mathrm{F}}^{\mathrm{b}}(\mathscr{A}) / \mathrm{K}^{\mathrm{b}}(\mathcal{P}(F))$. Take a minimal $F$-injective resolution of $\operatorname{Im}\left(d_{X}^{-r-2}\right)$ :

$$
0 \rightarrow \operatorname{Im}\left(d_{X}^{-r-2}\right) \rightarrow I^{0} \rightarrow I^{1} \rightarrow \cdots \rightarrow I^{r+1} \rightarrow M \rightarrow 0
$$

such that $M \simeq \Omega_{F}^{-r-2}\left(I m d_{X}^{-r-2}\right)$, where $I^{i}$ are $F$-injectives for $0 \leq i \leq r+1$. Then $\operatorname{Im}\left(d_{X}^{-r-2}\right)[r+2] \simeq M$ in $\mathrm{D}_{\mathrm{F}}^{\mathrm{b}}(\mathscr{A}) / \mathrm{K}^{\mathrm{b}}(\mathscr{P}(F))$. Therefore, $L(M) \simeq X^{\bullet}$. This shows that $L$ is dense.

Remark. In [20], Grime studied the special closed subfunctor $F$ of $\operatorname{Ext}^{1}(-,-)$. We generalize his main result to general closed subfunctor $F$.

Example. The Artin algebra $\Lambda$ is said to be an $F$-Frobenuis algebra if $\mathcal{P}(F)=I(F)$. Set $\mathscr{A}=\Lambda$-mod. Then we have a triangle equivalence $\Lambda-\bmod / \mathcal{P}(F) \simeq \mathrm{D}_{\mathrm{F}}^{\mathrm{b}}(\Lambda) / \mathrm{K}^{\mathrm{b}}(\mathcal{P}(F))$ by Theorem 5.1.

As a corollary of Theorem 5.1, we re-obtain Rickard's result.

Corollary 5.2. 41, Theorem 2.1] Let $\Lambda$ be a self-injective Artin algebra. Then we have the following triangle equivalence

$$
\mathrm{D}^{\mathrm{b}}(\Lambda-\bmod ) / \mathrm{K}^{\mathrm{b}}\left({ }_{\Lambda} \mathcal{P}\right) \simeq \Lambda \text { - } \underline{\bmod } .
$$

\section{Relative derived equivalences for Artin algebras}

In this section, suppose $\Lambda$ is an Artin $R$-algebras. Let $F$ be a non-zero subfunctor of $\operatorname{Ext}_{\Lambda}^{1}(-,-)$. Let $\Lambda$-mod be the category of finitely generated left $\Lambda$-modules. Denote by $\mathcal{P}(F)$ the subcategory of $\Lambda$-mod consisting of all $F$-projective $\Lambda$-modules. The subcategory ${ }_{\Lambda} \mathcal{P}$ of finitely generated projective $\Lambda$-modules is contained in $\mathcal{P}(F)$. Assume that $F$ has enough projectives and injectives, such that there exists $G \in \Lambda^{-}$ mod such that add $G=\mathcal{P}(F)$. Let $\mathrm{D}_{\mathrm{F}}^{\mathrm{b}}(\Lambda)$ be the relative bounded derived category of $\Lambda$-mod.

We give our main result in this section, a Morita theory for relative derived categories.

Theorem 6.1. Let $\Lambda$ and $\Gamma$ be Artin algebras. The following conditions are equivalent.

(1) $\mathrm{D}_{\mathrm{F}}^{\mathrm{b}}(\Lambda)$ and $\mathrm{D}^{\mathrm{b}}(\Gamma)$ are equivalent as triangulated categories.

(2) $\mathrm{K}^{\mathrm{b}}(\mathcal{P}(F))$ and $\mathrm{K}^{\mathrm{b}}\left(\Gamma_{\Gamma} \mathcal{P}\right)$ are equivalent as triangulated categories.

(3) $\mathrm{K}^{-}(\mathcal{P}(F))$ and $\mathrm{K}^{-}\left(\Gamma_{\Gamma} \mathcal{P}\right)$ are equivalent as triangulated categories.

(4) $\Gamma$ is isomorphic to $\operatorname{End}\left(T^{\bullet}\right)$, where $T^{\bullet}$ is an object of $\mathrm{K}^{\mathrm{b}}(\mathcal{P}(F))$ satisfying

(i) $\operatorname{Hom}\left(T^{\bullet}, T^{\bullet}[i]\right)=0$ for $i \neq 0$.

(ii) $\operatorname{add}\left(T^{\bullet}\right)$, the category of direct summands of finite direct sums of copies of $T^{\bullet}$, generates $\mathrm{K}^{\mathrm{b}}(\mathcal{P}(F))$ as a triangulated category.

Proof. There exists a $\Lambda$-module $G$ such that add $G=\mathscr{P}(F)$. Let $\Sigma=\operatorname{End}_{\Lambda}(G)$. Since

$$
\operatorname{Hom}_{\Lambda}(G,-): \operatorname{add} G \rightarrow_{\Sigma} \mathcal{P}
$$

is an equivalence, it follows that $\mathrm{K}^{-, F b}(\mathcal{P}(F))$ and $\mathrm{K}^{-, b}\left({ }_{\Sigma} \mathcal{P}\right)$ are equivalent as triangulated categories by the functor $\operatorname{Hom}_{\Lambda}(G,-)$, where $\mathrm{K}^{-, F b}(\mathcal{P}(F))=\left\{X^{\bullet} \in \mathrm{K}^{-}(\mathcal{P}(F)) \mid \exists n \in\right.$ $\mathbb{Z}$ such that $H^{i}\left(\operatorname{Hom}_{A}\left(G, X^{\bullet}\right)\right)=0$ for $\left.i \leq n\right\}$. We want to show that $\mathrm{D}^{\mathrm{b}}(\Sigma)$ and $\mathrm{D}^{\mathrm{b}}(\Gamma)$ are triangle equivalent. It suffices to prove that $\operatorname{Hom}_{\Lambda}\left(G, T^{\bullet}\right)$ is an ordinary tilting complex over $\Sigma$.

(i) $\operatorname{Hom}_{\Lambda}\left(G, T^{\bullet}\right) \in \mathrm{K}^{\mathrm{b}}\left({ }_{\Sigma} \mathcal{P}\right)$.

(ii) $\operatorname{Hom}\left(\left(G, T^{\bullet}\right),\left(G, T^{\bullet}\right)[i]\right) \simeq \operatorname{Hom}(T, T[i])=0$ fori $\neq 0$.

(iii) add $\left(\operatorname{Hom}\left(G, T^{\bullet}\right)\right)$ generates $\mathrm{K}^{\mathrm{b}}(\Sigma \mathcal{P})$ as triangulated category.

Finally,

$$
\operatorname{Hom}\left(\left(G, T^{\bullet}\right),\left(G, T^{\bullet}\right)\right) \simeq \operatorname{Hom}\left(G \otimes_{\Sigma}\left(G, T^{\bullet}\right), T^{\bullet}\right) \simeq \operatorname{Hom}\left(T^{\bullet}, T^{\bullet}\right)=\Gamma .
$$

Then by [40, Theorem 6.4], $\mathrm{D}^{\mathrm{b}}(\Sigma)$ and $\mathrm{D}^{\mathrm{b}}(\Gamma)$ are equivalent as triangulated categories.

Remark. If $F=\operatorname{Ext}^{1}(-,-)$, then we get Rickard's result [40, Corollary 8.3].

Definition 6.2. If $\Lambda$ and $\Gamma$ satisfy the equivalent conditions of Theorem 6.1, then $\Lambda$ and $\Gamma$ are said to be relatively derived equivalent. 
We shall call an object $T^{\bullet}$ of $\mathrm{K}^{\mathrm{b}}(\mathcal{P}(F))$ satisfying conditions (i) and (ii) of Theorem 6.1(4) an $F$ (or a relative)-tilting complex for $\Lambda$.

Next, we give an example of a relative tilting complex.

Example. Let $k$ be a field and $\Lambda$ the algebra given by the quiver

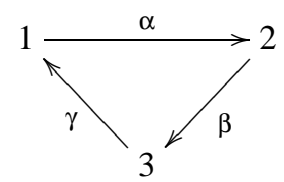

and relations $\alpha \beta \gamma=\beta \gamma \alpha \beta=\gamma \alpha \beta \gamma=0$.

Let $P_{1}, P_{2}, P_{3}$ be indecomposable projective $\Lambda$-modules associated with vertices $1,2,3$, respectively. Let $F=F_{\mathcal{P}(F)}$ be the subbifunctor with $\mathcal{P}(F)=\operatorname{add}\left(P_{1} \oplus P_{2} \oplus P_{3} \oplus M\right)$, where $M=\operatorname{rad}\left(P_{1}\right)$. Let $T_{1}^{\bullet}$ be the complex $0 \rightarrow Q \stackrel{f}{\rightarrow} M \oplus P_{1} \rightarrow 0$, where $f$ is an $\operatorname{add}\left(P_{2} \oplus P_{3}\right)$-approximation of $\left(M \oplus P_{1}\right)$ and $Q \in \operatorname{add}\left(P_{2} \oplus P_{3}\right)$ is in degree -1 . Let $T_{2}^{\bullet}: 0 \rightarrow P_{2} \oplus P_{3} \rightarrow 0$ be a stalk complex concentrated in degree -1 . In this relative theory, $T^{\bullet}=T_{1}^{\bullet} \oplus T_{2}^{\bullet}$ is an $F$-tilting complex over $\Lambda$. This has been showed in [26, Proposition 1.2] and [28, Theorem 3.5]. There is a distinguished triangle

$$
Q[-1] \rightarrow\left(M \oplus P_{1}\right)[1] \rightarrow T_{1}^{\bullet} \rightarrow Q
$$

Then $G=P_{1} \oplus P_{2} \oplus P_{3} \oplus M \in \operatorname{add} T^{\bullet}$. By the proof of Theorem 6.1, it follows that $\operatorname{Hom}\left(G, T^{\bullet}\right)$ is a tilting complex for $\operatorname{End}(G)$.

As for the theory of derived equivalence, it is useful to study the Grothendieck groups in order to determined the number of indecomposable non-isomorphic summands which a tilting complex has. We introduce the following group. Let $\mathbb{Z}(\Lambda$-mod $)$ denote the free abelian group with the isomorphism classes $[X]$ of $\Lambda$-modules $X$. Define the $F$-stable Grothendieck group of $\Lambda$-mod, $F-K_{0}(\Lambda$-mod), to be $\mathbb{Z}(\Lambda$ mod $) / F-R(\Lambda$-mod $)$, where $F-R(\Lambda$-mod $)$ is the subgroup of $\mathbb{Z}(\Lambda$-mod $)$ generated by the elements $[X]+$ $[Z]-[Y]$ whenever $0 \rightarrow X \rightarrow Y \rightarrow Z \rightarrow 0$ is an $F$-exact sequence of $\Lambda$-modules.

Proposition 6.3. Let $\Lambda$ and $\Gamma$ be relatively derived equivalent. Then

$$
F-K_{0}(\Lambda-\bmod ) \simeq K_{0}(\Gamma-\bmod ) .
$$

Proof. If $\Lambda$ and $\Gamma$ are relatively derived equivalent, then $K_{0}\left(\mathrm{D}_{\mathrm{F}}^{\mathrm{b}}(\Lambda)\right) \simeq K_{0}\left(\mathrm{D}^{\mathrm{b}}(\Gamma)\right)$. As is known, $K_{0}(\Gamma-$ mod $) \simeq K_{0}\left(\mathrm{D}^{\mathrm{b}}(\Gamma)\right)$. It suffices to show that $F-K_{0}(\Lambda-\bmod ) \simeq K_{0}\left(\mathrm{D}_{\mathrm{F}}^{\mathrm{b}}(\Lambda)\right)$.

Define a map $\alpha: F-K_{0}(\Lambda-\bmod ) \rightarrow K_{0}\left(\mathrm{D}_{\mathrm{F}}^{\mathrm{b}}(\Lambda)\right)$ by $\alpha([X])=[l(X)]$, where $l: \Lambda$-mod $\rightarrow \mathrm{D}^{\mathrm{b}}(\Gamma)$ is the inclusion functor.

Suppose that $X \rightarrow Y \rightarrow Z \rightarrow X[1]$ is a distinguished triangle in $\mathrm{D}_{\mathrm{F}}^{\mathrm{b}}(\Lambda)$ and $P \in \mathcal{P}(F)$. Applying the functor $\operatorname{Hom}_{\mathrm{D}_{\mathrm{F}}^{\mathrm{b}}(\Lambda)}(P,-)$ to the above triangle, we have a exact sequence

$$
0 \rightarrow(P, X) \rightarrow(P, Y) \rightarrow(P, Z) \rightarrow 0
$$

It follows that $0 \rightarrow X \rightarrow Y \rightarrow Z \rightarrow 0$ is an $F$-exact sequence in $\Lambda$-mod. Then we define $\beta: K_{0}\left(\mathrm{D}_{\mathrm{F}}^{\mathrm{b}}(\Lambda)\right) \rightarrow F$ $K_{0}\left(\Lambda\right.$-mod) by $\beta\left(\left[X^{\bullet}\right]\right)=\Sigma_{i}(-1)^{i}\left[\left(X^{i}\right)\right]$, where $X^{\bullet} \in \mathrm{D}_{\mathrm{F}}^{\mathrm{b}}(\Gamma)$. It is easy to show that $\alpha \beta=i d_{K_{0}\left(\mathrm{D}_{\mathrm{F}}^{\mathrm{b}}(\Lambda)\right)}$ and $\beta \alpha=i d_{F-K_{0}(\Lambda-m o d)}$.

As a consequence of Proposition 6.3 we have the following proposition.

Proposition 6.4. Let $T^{\bullet}$ be an $F$-tilting complex and $\Gamma=\operatorname{End}\left(T^{\bullet}\right)$. Then the following conditions are equivalent.

(1) The number of non-isomorphic indecomposable summands of $T^{\bullet}$.

(2) The number of non-isomorphic simple $\Gamma$-modules.

(3) The number of non-isomorphic indecomposable modules in $\mathcal{P}(F)$.

By J.Rickard's criterion [40, Proposition 6.2], we give a characterization of $\mathrm{K}^{\mathrm{b}}(I(F))$ which is a full triangulated subcategory of $\mathrm{D}_{\mathrm{F}}^{\mathrm{b}}(\Lambda)$.

Lemma 6.5. $\mathrm{K}^{\mathrm{b}}(I(F))=\left\{X^{\bullet} \in \mathrm{D}_{\mathrm{F}}^{\mathrm{b}}(\Lambda) \mid\right.$ for any $Y^{\bullet} \in \mathrm{D}_{\mathrm{F}}^{\mathrm{b}}(\Lambda)$, there exists $n_{0} \in \mathbb{Z}$, such that $\left.\operatorname{Hom}_{\mathrm{D}_{\mathrm{F}}^{\mathrm{b}}(\Lambda)}\left(Y^{\bullet}, X^{\bullet}[i]\right)=0, \forall i \geq n_{0}\right\}$. 
Proof. If $X^{\bullet} \in \mathrm{D}_{\mathrm{F}}^{\mathrm{b}}(\Lambda)$ and $X^{\bullet} \simeq I_{X}^{\bullet}$ with $I_{X}^{\bullet} \in \mathrm{K}^{\mathrm{b}}(I(F))$. Then

$$
\operatorname{Hom}_{\mathrm{D}_{\mathrm{F}}^{\mathrm{b}}(\Lambda)}\left(Y^{\bullet}, X^{\bullet}[i]\right) \simeq \operatorname{Hom}_{\mathrm{D}_{\mathrm{F}}^{\mathrm{b}}(\Lambda)}\left(Y^{\bullet}, I_{X}^{\bullet}[i]\right) \simeq \operatorname{Hom}_{\mathrm{K}^{\mathrm{b}}(\Lambda)}\left(Y^{\bullet}, I_{X}^{\bullet}[i]\right)=0
$$

for all $i \geq n_{0}$ with some $n_{0} \in \mathbb{Z}$.

If $X^{\bullet} \in \mathrm{D}_{\mathrm{F}}^{\mathrm{b}}(\Lambda)$ and for any $Y^{\bullet} \in \mathrm{D}_{\mathrm{F}}^{\mathrm{b}}(\Lambda), \exists n_{0} \in \mathbb{Z}$, such that $\operatorname{Hom}_{\mathrm{D}_{\mathrm{F}}^{\mathrm{b}}(\Lambda)}\left(Y^{\bullet}, X^{\bullet}[i]\right)=0$, for all $i \geq n_{0}$. Since $\mathrm{D}_{\mathrm{F}}^{\mathrm{b}}(\Lambda) \simeq \mathrm{K}^{+, b}(I(F))$, it follows that $X^{\bullet} \simeq I_{X}^{\bullet}$ with $I_{X}^{\bullet} \in K^{+, b}(I(F))$ such that $H^{i}\left(P, I_{X}^{\bullet}\right)=0$ for $i \geq m, m \in \mathbb{Z}$. For $j \geq m$, we have the following diagram

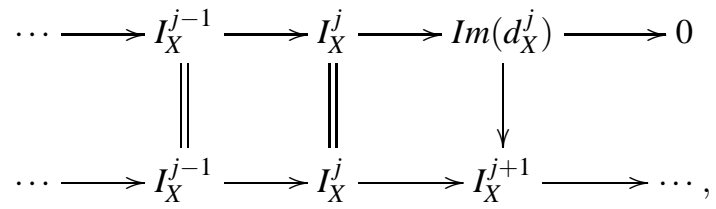

such that the complex $I_{X}^{\bullet}$ is isomorphic in $\mathrm{D}_{\mathrm{F}}^{\mathrm{b}}(\Lambda)$ to a complex of the form $\cdots \rightarrow I_{X}^{j-1} \rightarrow I_{X}^{j} \rightarrow \operatorname{Im}\left(d_{X}^{j}\right) \rightarrow 0$. We will prove that $\operatorname{Im}\left(d_{X}^{j}\right)$ is $F$-injective. If $\operatorname{Im}\left(d_{X}^{j}\right)$ is not $F$-injective, then there is $j=i-1 \geq m$ and $\operatorname{Im}\left(d_{X}^{i-1}\right) \neq 0$. Hence, the map $I_{X}^{i-1} \rightarrow \operatorname{Im}\left(d_{X}^{i-1}\right)$ is not zero. Set $Y=\oplus_{j \geq m} \operatorname{Im}\left(d_{X}^{j}\right)$. Then the map $I_{X}^{i-1} \rightarrow Y=\operatorname{Im}\left(d_{X}^{i-1}\right) \oplus_{j \geq m, j \neq i-1} \operatorname{Im}\left(d_{X}^{j}\right)$ is not zero. Consequently, $\operatorname{Hom}_{\mathrm{D}_{\mathrm{F}}^{\mathrm{b}}(\Lambda)}\left(Y, X^{\bullet}[i]\right) \neq 0$ for $i \geq m+1$. This leads to a contradiction with our assumption.

\section{Relative derived equivalences and relative homological dimen- sions}

In this section,we study the relationships between relative dimensions and relative derived equivalences.

Let $\Lambda$ be an Artin algebra. Let us recall some notions of relative homological dimensions. The $F$ projective dimension of a $\Lambda$-module $X$, denoted by proj. $\operatorname{dim}_{F} X$, is defined to be the smallest $m$, such that there is an $F$-projective resolution

$$
0 \rightarrow P^{-m} \rightarrow \cdots \rightarrow P^{-1} \rightarrow P^{0} \rightarrow X \rightarrow 0
$$

with $P^{-i} \in \mathcal{P}(F)$ for $i \geq 0$. If such $m$ does not exist, then define proj. $\operatorname{dim}_{F} X=\infty$. The $F$-global dimension of $\Lambda$ is defined as

$$
\operatorname{gl\cdot } \operatorname{dim}_{F}(\Lambda)=\sup \left\{\operatorname{proj} \cdot \operatorname{dim}_{F}(X) \mid X \in \Lambda \text {-mod }\right\} .
$$

The $F$-finitistic dimension of $\Lambda$ is defined as follows

$$
\text { fin. } \operatorname{dim}_{F}(\Lambda)=\sup \left\{\text { proj.dim } \operatorname{dim}_{F} X \mid \text { proj.dim } \operatorname{dim}_{F}(X)<\infty, X \in \Lambda \text {-mod }\right\} .
$$

The relative finitistic dimension rel.fin.dim $\operatorname{dim}_{F}(\Lambda)$ is defined to be $\sup _{F}$ fin.dim $\operatorname{dim}_{F}(\Lambda)$, where $F$ ranges over all subfunctors $F=F_{\mathscr{C}}$ for all subcategories of finite type of $\Lambda$-mod contains $\mathcal{P}(\Lambda)$.

We begin with the characterization of $F$-projective dimension of a $\Lambda$-module.

Lemma 7.1. The following conditions are equivalent.

(i) proj.dim $\operatorname{dim}_{F}(X) \leq m$.

(ii) $\operatorname{Ext}_{F}^{i}(X, Y)=0$ for all modules $Y$ and for all $i \geq m+1$.

(iii) $\operatorname{Ext}_{F}^{m+1}(X, Y)=0$ for all modules $Y$.

Recall that $\Lambda$ and $\Gamma$ are relatively derived equivalent, if there is a triangle equivalence $\mathrm{D}_{\mathrm{F}}^{\mathrm{b}}(\Lambda) \simeq \mathrm{D}^{\mathrm{b}}(\Gamma)$, where $\Gamma \simeq \operatorname{End}\left(T^{\bullet}\right), T^{\bullet}$ is a relative tilting complex for $\Lambda$. Clearly, $T^{\bullet}$ is a bounded complex. Let $n \geq 0$ be an integer. Without loss of generality, we may assume that $T^{\bullet}$ is a radical complex of the form:

$$
\cdots \rightarrow 0 \rightarrow T^{-n} \rightarrow T^{-n+1} \rightarrow \cdots \rightarrow T^{-1} \rightarrow T^{0} \rightarrow 0 \rightarrow \cdots .
$$

The integer $n$ is called the term length of $T^{\bullet}$, denote by $t\left(T^{\bullet}\right)=n$.

Let $H: \mathrm{D}^{\mathrm{b}}(\Gamma) \rightarrow \mathrm{D}_{\mathrm{F}}^{\mathrm{b}}(\Lambda)$ be a relative derived equivalence such that $H(\Gamma)=T^{\bullet}$. Then we have the following fact. 
Lemma 7.2. Let $n \geq 0$ be an integer and $T^{\bullet}$ as above. Let $L: \mathrm{D}_{\mathrm{F}}^{\mathrm{b}}(\Lambda) \rightarrow \mathrm{D}^{\mathrm{b}}(\Gamma)$ be a quasi-inverse of $H$. Then there exists a radical tilting complex $\bar{T}^{\bullet} \in \mathrm{K}^{\mathrm{b}}\left({ }_{\Gamma} \mathcal{P}\right)$ such that $L(G)=\bar{T}^{\bullet}$ and $\bar{T}^{i}=0$ for $i>n$ or $i<0$.

Proof. Considering the homological group of $\bar{T}$, we have

$$
H^{i}\left(\bar{T}^{\bullet}\right) \simeq \operatorname{Hom}_{\mathrm{K}^{\mathrm{b}}(\Gamma)}\left(\Gamma, \bar{T}^{\bullet}[i]\right) \simeq \operatorname{Hom}_{\mathrm{D}^{\mathrm{b}}(\Gamma)}\left(\Gamma, \bar{T}^{\bullet}[i]\right) \simeq \operatorname{Hom}_{\mathrm{D}_{\mathrm{F}}^{\mathrm{b}}(\Lambda)}\left(T^{\bullet}, G[i]\right) .
$$

By Proposition 3.7 we have $\operatorname{Hom}_{\mathrm{D}_{\mathrm{F}}^{\mathrm{b}}(\Lambda)}\left(T^{\bullet}, G[i]\right) \simeq \operatorname{Hom}_{\mathrm{K}^{\mathrm{b}}(\Lambda)}\left(T^{\bullet}, G[i]\right)$. Then $H^{i}\left(\bar{T}^{\bullet}\right)=0$ for $i>n$ or $i<0$ by transferring shifts. Since $\bar{T}^{\bullet} \in \mathrm{K}^{\mathrm{b}}\left({ }_{\Gamma} \mathcal{P}\right)$, it follows that $\bar{T}^{\bullet}$ has the following form

$$
\cdots \rightarrow 0 \rightarrow \bar{T}^{-r} \rightarrow \bar{T}^{-r+1} \rightarrow \cdots \rightarrow \bar{T}^{-1} \stackrel{d^{-1}}{\rightarrow} \bar{T}^{0} \stackrel{d^{0}}{\rightarrow} \bar{T}^{1} \rightarrow \bar{T}^{s} \rightarrow 0 \rightarrow \cdots
$$

where $r, s \in \mathbb{N}$. If $H^{i}\left(\bar{T}^{\bullet}\right)=0$ for $i>n$, then the following sequence

$$
0 \rightarrow \operatorname{Im}\left(d_{T}^{n}\right) \rightarrow \bar{T}^{n+1} \rightarrow \cdots \rightarrow \bar{T}^{s} \rightarrow 0 \rightarrow \cdots
$$

is a split exact sequence. Consequently, $\operatorname{Im}\left(d_{T}^{n}\right)$ is a projective $\Gamma$-module. Then the complex $\bar{T}^{\bullet}$ is isomorphic in $\mathrm{D}^{\mathrm{b}}(\Gamma)$ to a complex of the form

$$
0 \rightarrow \bar{T}^{-r} \rightarrow \bar{T}^{-r+1} \rightarrow \cdots \rightarrow \bar{T}^{-1} \rightarrow \bar{T}^{0} \rightarrow \cdots \rightarrow \bar{T}^{n-1} \rightarrow \operatorname{Ker}\left(d_{T}^{n}\right) \rightarrow 0,
$$

where $\operatorname{Ker}\left(d_{T}^{n}\right)$ is a projective $\Gamma$-module. On the other hand, we have

$$
H^{i}\left(\operatorname{Hom}_{\Gamma}\left(\bar{T}^{\bullet}, \Gamma\right)\right) \simeq \operatorname{Hom}_{\mathrm{D}^{\mathrm{b}}(\Gamma)}\left(\bar{T}^{\bullet}, \Gamma[i]\right) \simeq \operatorname{Hom}_{\mathrm{D}_{\mathrm{F}}^{\mathrm{b}}(\Lambda)}\left(G, T^{\bullet}[i]\right) \simeq \operatorname{Hom}_{\mathrm{K}^{\mathrm{b}}(\Lambda)}\left(G, T^{\bullet}[i]\right)=0 .
$$

for $i>0$. It follows that

$$
0 \rightarrow \operatorname{Im}\left(d_{T}^{0}, \Gamma\right) \rightarrow\left(\bar{T}^{-1}, \Gamma\right) \rightarrow\left(\bar{T}^{-2}, \Gamma\right) \rightarrow \cdots
$$

is split exact in $\mathrm{K}^{\mathrm{b}}\left(\Gamma^{o p}\right)$, where $\operatorname{Im}\left(d_{T}^{0}, \Gamma\right)=\left(\operatorname{Im}\left(d_{T}^{-1}\right), \Gamma\right)$. We conclude that $\operatorname{Ker}\left(d_{T}^{0}, \Gamma\right)=$ $\left(\operatorname{Coker}\left(d_{T}^{-1}\right), \Gamma\right)$ is a projective $\Gamma$-module. Consequently, $\operatorname{Coker}\left(d_{T}^{-1}\right)$ and $\operatorname{Im}\left(d_{T}^{-1}\right)$ are projective $\Gamma$ modules. Therefore, the complex $\bar{T}^{\bullet}$ is isomorphic a complex of the following form

$$
\cdots \rightarrow 0 \rightarrow \operatorname{Coker}\left(d_{T}^{-1}\right) \rightarrow \bar{T}^{1} \rightarrow \cdots \rightarrow \bar{T}^{n-1} \rightarrow \operatorname{Ker}\left(d_{T}^{n}\right) \rightarrow 0 \quad \text { in } \quad \mathrm{K}^{\mathrm{b}}(\Gamma \mathcal{P}) .
$$

This completes the proof.

The main result of this section can be stated as follows.

Theorem 7.3. Let $L: \mathrm{D}_{\mathrm{F}}^{\mathrm{b}}(\Lambda) \rightarrow \mathrm{D}^{\mathrm{b}}(\Gamma)$ be a relative derived equivalence. Suppose $T^{\bullet}$ is the relative tilting complex associated to $L$. Then we have

(1) $\operatorname{gl} \cdot \operatorname{dim}_{F}(\Lambda)-t\left(T^{\bullet}\right) \leq$ gl.dim $(\Gamma) \leq \operatorname{gl} \cdot \operatorname{dim}_{F}(\Lambda)+t\left(T^{\bullet}\right)+2$.

(2) fin.dim $\operatorname{dim}_{F}(\Lambda)-t\left(T^{\bullet}\right) \leq$ fin.dim $(\Gamma) \leq$ fin.dim $\operatorname{dim}_{F}(\Lambda)+t\left(T^{\bullet}\right)+2$.

The following lemmas are useful in our proofs.

Lemma 7.4. Let $Y$ be a $\Gamma$-module. Then $H^{i}(H(Y))=0$ for $i<-n$ or $i>0$, and in $\mathrm{D}_{\mathrm{F}}^{\mathrm{b}}(\Lambda), H(Y)$ is isomorphic to a complex $T_{Y}^{\bullet}$ of the following form

$$
\cdots \rightarrow 0 \rightarrow \operatorname{Im}\left(d_{T_{Y}}^{-n-2}\right) \rightarrow T_{Y}^{-n-1} \rightarrow \cdots \rightarrow T_{Y}^{-1} \rightarrow \operatorname{Ker}\left(d_{T_{Y}}^{0}\right) \rightarrow 0 \rightarrow \cdots,
$$

where $T_{Y}^{i}$ and $\operatorname{Ker}\left(d_{Y}^{0}\right)$ are $F$-projective $\Lambda$-modules.

Proof. We have the following isomorphisms

$$
H^{i}(H(Y)) \simeq \operatorname{Hom}_{\mathrm{D}_{\mathrm{F}}^{\mathrm{b}}(\Lambda)}(\Lambda, H(Y)[i]) \simeq \operatorname{Hom}_{\mathrm{D}^{\mathrm{b}}(\Gamma)}\left(\bar{T}_{\Lambda}^{\bullet}, Y[i]\right) \simeq \operatorname{Hom}_{\mathrm{K}(\Gamma)}\left(\bar{T}_{\Lambda}^{\bullet}, Y[i]\right),
$$

where $L(\Lambda) \simeq \bar{T}_{\Lambda}^{\bullet}$ has the same form as $\bar{T}^{\bullet}$. Then $H^{i}(H(Y))=0$ for $i>0$ or $i<-n$. Assume that $H(Y)$ is isomorphic to a bounded above $F$-projective complex:

$$
\cdots \rightarrow T_{Y}^{-n-1} \rightarrow T_{Y}^{-n} \rightarrow \cdots \rightarrow T_{Y}^{-1} \rightarrow T_{Y}^{0} \rightarrow \cdots \rightarrow T_{Y}^{S} \rightarrow 0
$$


Then we get two acyclic complexes

$$
\cdots \rightarrow T_{Y}^{-n-1} \rightarrow \operatorname{Im}\left(d_{T_{Y}}^{-n-1}\right) \rightarrow 0 \rightarrow \cdots
$$

and

$$
0 \rightarrow \operatorname{Im}\left(d_{T_{Y}}^{0}\right) \rightarrow T_{Y}^{1} \rightarrow \cdots
$$

For any $P \in \mathcal{P}(F)$, we have

$$
H^{i}(P, H(Y)) \simeq \operatorname{Hom}_{\mathrm{D}_{\mathrm{F}}^{\mathrm{b}}(\Lambda)}(P, H(Y)[i]) \simeq \operatorname{Hom}_{\mathrm{D}^{\mathrm{b}}(\Gamma)}\left(\bar{T}_{P}^{\bullet}, Y[i]\right) \simeq H^{i}\left(\operatorname{Hom}_{\Gamma}\left(\bar{T}_{P}^{\bullet}, Y\right)\right)=0
$$

for $i>0$ or $i<-n$, where $L(P) \simeq \bar{T}_{P}^{\bullet}$ has the same form as $\bar{T}^{\bullet}$. Therefore, we get the following acyclic complexes:

$$
\cdots \rightarrow \operatorname{Hom}\left(P, T_{Y}^{-n-1}\right) \rightarrow \operatorname{Im} \operatorname{Hom}\left(P, d_{T_{Y}}^{-n-1}\right) \rightarrow 0
$$

and

$$
0 \rightarrow \operatorname{Im}\left(\operatorname{Hom}\left(P, d_{T_{Y}}^{0}\right)\right) \rightarrow \operatorname{Hom}\left(P, T_{Y}^{1}\right) \rightarrow \cdots .
$$

We conclude that there exist two $F$-acyclic complexes:

$$
\cdots \rightarrow T_{Y}^{-n-2} \rightarrow \operatorname{Im}\left(d_{T_{Y}}^{-n-2}\right) \rightarrow 0 \rightarrow \cdots
$$

and

$$
0 \rightarrow \operatorname{Im}\left(d_{T_{Y}}^{0}\right) \rightarrow T_{Y}^{1} \rightarrow \cdots .
$$

Then the complex $H(Y)$ is isomorphic in $\mathrm{D}_{\mathrm{F}}^{\mathrm{b}}(\Lambda)$ to a complex $T_{Y}^{\bullet}$ of the following form:

$$
\cdots \rightarrow 0 \rightarrow \operatorname{Im}\left(d_{T_{Y}}^{-n-2}\right) \rightarrow T_{Y}^{-n-1} \rightarrow \cdots \rightarrow T_{Y}^{-1} \rightarrow \operatorname{Ker}\left(d_{T_{Y}}^{0}\right) \rightarrow 0 \rightarrow \cdots,
$$

where $\operatorname{Ker}\left(d_{T_{Y}}^{0}\right)$ and $T_{Y}^{-i}(i=0, \cdots, n-1)$ are $F$-projective $\Lambda$-modules.

Lemma 7.5. Let $X$ be a $\Lambda$-module. Then $H^{i}(L(X))=0$ for $i>n$ or $i<0$, and in $\mathrm{D}^{\mathrm{b}}(\Gamma), L(X)$ is isomorphic to a complex $\bar{T}_{X}^{\bullet}$ of the following form:

$$
\cdots \rightarrow 0 \rightarrow \operatorname{Coker}\left(d_{T_{X}}^{-1}\right) \rightarrow \bar{T}_{X}^{1} \rightarrow \cdots \rightarrow \bar{T}_{X}^{n-1} \rightarrow \operatorname{Ker}\left(d_{T_{X}}^{n}\right) \rightarrow 0 \rightarrow \cdots
$$

where $\bar{T}_{X}^{i}(1 \leq i \leq n-1)$ and $\operatorname{Kerd}^{n}$ are projective $\Gamma$-modules.

Lemma 7.6. 38, Lemma3.7] Let $m, t, d \in \mathbb{N}, X^{\bullet}, Y^{\bullet} \in \mathrm{K}^{\mathrm{b}}(\Lambda$-mod $)$. Assume that $X^{i}=0$ for $i<m, Y^{j}=0$ for $j>t$, and $\operatorname{Ext}_{F}^{l}\left(X^{i}, Y^{j}\right)=0$ for all $i, j \in \mathbb{N}$ and $l \geq d$. Then $\operatorname{Hom}_{\mathrm{D}_{\mathrm{F}}^{\mathrm{b}}(\Lambda)}\left(X^{\bullet}, Y^{\bullet}[l]\right)=0$ for $l \geq d+t-m$.

Proof. The proof is similar to the one in [32, Lemma 1.6]. The detailed proofs will appear in [38].

We now have all the ingredients to complete the proof of our main theorem.

\section{Proof of Theorem 7.3:}

Assume that $\operatorname{fin}_{\operatorname{dim}}(\Lambda)<\infty$. Let $N$ be arbitrary $\Gamma$-module, and let $M$ be a $\Gamma$-module with proj. $\operatorname{dim}_{\Gamma}(M)<\infty$. Let $P_{M}^{\bullet} \rightarrow M$ be a minimal projective resolution of $M$. We have the following isomorphisms

$$
\operatorname{Ext}_{\Gamma}^{i}(M, N) \simeq \operatorname{Hom}_{\mathrm{D}^{\mathrm{b}}(\Gamma)}(M, N[i]) \simeq \operatorname{Hom}_{\mathrm{D}^{\mathrm{b}}(\Gamma)}\left(P_{M}^{\bullet}, N[i]\right) \simeq \operatorname{Hom}_{\mathrm{D}_{\mathrm{F}}^{\mathrm{b}}(\Lambda)}\left(H\left(P_{M}^{\bullet}\right), H(N)[i]\right) .
$$

By Lemma 7.4, we see that $H(N)$ is isomorphic in $\mathrm{D}_{\mathrm{F}}^{\mathrm{b}}(\Lambda)$ to a complex $T_{N}^{\bullet}$ of the form:

$$
\cdots \rightarrow 0 \rightarrow \operatorname{Im}\left(d_{T_{N}}^{-n-2}\right) \rightarrow T_{N}^{-n-1} \rightarrow \cdots \rightarrow T_{N}^{-1} \rightarrow \operatorname{Ker}\left(d_{T_{N}}^{0}\right) \rightarrow 0 \rightarrow \cdots .
$$

By Theorem 6.1, it follows that $H\left(P_{M}^{\bullet}\right) \simeq T_{M}^{\bullet} \in \mathrm{K}^{\mathrm{b}}(\mathcal{P}(F))$ and $H^{i}\left(H\left(P_{M}^{\bullet}\right)\right)=0$ for $i<-n$ or $i>0$. Then we see that

$$
0 \rightarrow T_{M}^{-r} \rightarrow \cdots \rightarrow \operatorname{Ker}\left(d_{T_{M}}^{-n-2}\right) \rightarrow 0
$$


and

$$
0 \rightarrow \operatorname{Im}\left(d_{T_{M}}^{0}\right) \rightarrow \cdots \rightarrow T_{M}^{s} \rightarrow 0
$$

are $F$-split acyclic complexes, where $\operatorname{Im}\left(d_{T_{M}}^{0}\right)$ is an $F$-projective $\Lambda$-module. Therefore, $T_{M}^{\bullet}$ is isomorphic to a complex of the following form

$$
0 \rightarrow \operatorname{Im}\left(d_{T_{M}}^{-n-2}\right) \rightarrow T_{M}^{-n} \rightarrow \cdots \rightarrow T_{M}^{-1} \rightarrow \operatorname{Ker}\left(d_{T_{M}}^{0}\right) \rightarrow 0 \quad \text { in } \quad \mathrm{D}_{\mathrm{F}}^{\mathrm{b}}(\Lambda),
$$

where $\operatorname{Ker}\left(d_{T_{M}}^{0}\right)$ and $T_{M}^{i}(-n \leq i \leq-1)$ are $F$-projective $\Lambda$-modules. From the above argument, we deduce that proj. $\operatorname{dim}_{F}\left(\operatorname{Im}\left(d_{T_{M}}^{-n-2}\right)\right)<\infty$. Consequently, proj.dim $\operatorname{dim}_{F}\left(\operatorname{Im}\left(d_{T_{M}}^{-n-2}\right)\right) \leq \operatorname{fin} \cdot \operatorname{dim}_{F}(\Lambda)$. By Lemma7.6, we conclude that

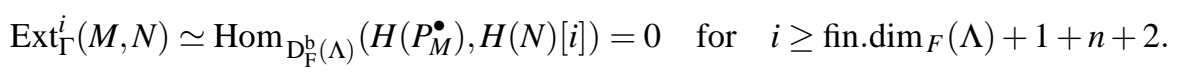

This implies that fin.dim $(\Gamma) \leq$ fin. $\operatorname{dim}_{F}(\Lambda)+l\left(T^{\bullet}\right)+2$.

Assume that fin.dim $(\Gamma)<\infty$. Let $Y$ be any $\Lambda$-module, and let $X$ be a $\Lambda$-module with proj.dim $\operatorname{dim}_{F}(X)<$ $\infty$. Let $P_{X}^{\bullet} \rightarrow X$ be a minimal $F$-projective resolution of $X$. We have the following isomorphisms

$$
\operatorname{Ext}_{F}^{i}(X, Y) \simeq \operatorname{Hom}_{\mathrm{D}_{\mathrm{F}}^{\mathrm{b}}(\Lambda)}(X, Y[i]) \simeq \operatorname{Hom}_{\mathrm{D}_{\mathrm{F}}^{\mathrm{b}}(\Lambda)}\left(P_{X}^{\bullet}, Y[i]\right) \simeq \operatorname{Hom}_{\mathrm{D}^{\mathrm{b}}(\Gamma)}\left(L\left(P_{X}^{\bullet}\right), L(Y)[i]\right) .
$$

By Lemma 7.5, we see that $L(Y)$ is isomorphic in $\mathrm{D}^{\mathrm{b}}(\Gamma)$ to a complex of the following form:

$$
\cdots \rightarrow 0 \rightarrow \operatorname{Coker}\left(d_{T_{Y}}^{-1}\right) \rightarrow \bar{T}_{Y}^{1} \rightarrow \cdots \rightarrow \bar{T}_{Y}^{n-1} \rightarrow \operatorname{Ker}\left(d_{T_{Y}}^{n}\right) \rightarrow 0 \rightarrow \cdots .
$$

From Theorem 6.1, it follows that $H\left(P_{X}^{\bullet}\right) \simeq \bar{T}_{X}^{\bullet} \in \mathrm{K}^{\mathrm{b}}\left({ }_{\Gamma} \mathcal{P}\right)$ and $H^{i}\left(L\left(P_{X}^{\bullet}\right)\right)=0$ for $i<0$ or $i>n$. Therefore, $\bar{T}_{X}^{\bullet}$ is isomorphic to a complex of the form

$$
\cdots \rightarrow 0 \rightarrow \operatorname{Coker}\left(d_{T_{Y}}^{-1}\right) \rightarrow \bar{T}_{X}^{1} \rightarrow \cdots \rightarrow \bar{T}_{X}^{n-1} \rightarrow \operatorname{Ker}\left(d_{T_{Y}}^{n}\right) \rightarrow 0 \rightarrow \cdots \quad \text { in } \quad \mathrm{D}^{\mathrm{b}}(\Gamma),
$$

where $\operatorname{Ker}\left(d_{T_{Y}}^{n}\right), T_{X}^{i}(1 \leq i \leq n)$ are projective $\Gamma$-modules. We see that proj.dim $\left(\operatorname{Coker}\left(d_{T_{Y}}^{-1}\right)\right)<\infty$. Consequently, proj.dim $\left(\operatorname{Coker}\left(d_{T_{Y}}^{-1}\right)\right) \leq \operatorname{fin} \cdot \operatorname{dim}(\Gamma)$. By Lemma 7.6, we conclude that

$$
\operatorname{Ext}_{F}^{i}(X, Y) \simeq \operatorname{Hom}_{\mathrm{D}^{\mathrm{b}}(\Gamma)}\left(L\left(P_{X}^{\bullet}\right), L(Y)[i]\right)=0 \quad \text { for } \quad i \geq \operatorname{fin} \cdot \operatorname{dim}(\Gamma)+1+n .
$$

This implies that fin.dim $\operatorname{dim}_{F}(\Lambda) \leq$ fin.dim $(\Gamma)+l\left(T^{\bullet}\right)$.

Remark. From Theorem 7.3, we see that if the relative finitistic dimension of $\Lambda$ is finite if and only if the finitistic dimension of $\Gamma$ is finite. Auslander and Solberg [3, Proposition 6.1] proved that the standard finitistic dimension conjecture is true for all Artin algebras if and only if the relative finitistic dimension conjecture is true. Then we get another approach to the finitistic dimension conjecture.

Example. The standard global dimension $g l \cdot \operatorname{dim}(\Lambda)$ can be infinite, but gl.dim $(\Gamma)$ is finite. A less trivial example is the following. Let $\Lambda$ be the path algebra of the following quiver

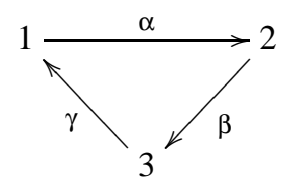

and relations $\alpha \beta \gamma=\beta \gamma \alpha=\gamma \alpha \beta=0$. Let $P_{1}=\frac{1}{2}, P_{2}=\frac{2}{3}$ and $P_{3}=\frac{3}{2}$, which are all the indecomposable projective $\Lambda$-modules. Then let $M_{i}=P_{i} / \operatorname{soc}\left(P_{i}\right)$ and $S_{i}=P_{i} / \operatorname{rad}\left(P_{i}\right)$ for $i=1,2,3$, where $\operatorname{soc}\left(P_{i}\right)$ is the socle of $P_{i}$ for each $i$. Let $\mathcal{P}(F)=\left\{P_{1}, P_{2}, P_{3}, S_{2}, S_{3}, M_{2}\right\}$. Then we have $I(F)=\mathrm{D} \operatorname{Tr}(\mathcal{P}(\mathrm{F})) \cup_{\Lambda} I=$ $\left\{\mathrm{P}_{1}, \mathrm{P}_{2}, \mathrm{P}_{3}, \mathrm{~S}_{3}, \mathrm{~S}_{1}, \mathrm{M}_{3}\right\}$, where $\mathrm{DTr}$ is the Auslander-Reiten translation of $\Lambda$. It is easy to find $F$-exact sequences for the modules $S_{1}, M_{1}$ and $M_{3}$ to show that $\operatorname{gldim}_{F}(\Lambda) \leq 1$. By Theorem 7.3 we have gl.dim $(\Gamma) \leq \operatorname{gl\cdot dim} F(\Lambda)+l\left(T^{\bullet}\right)+2$ for any $F$-tilting complex $T^{\bullet}$, where $\operatorname{End}\left(T^{\bullet}\right) \simeq \Gamma$. In particular, for the $F$-tilting module

$$
T^{\bullet}=0 \rightarrow P_{1} \oplus P_{2} \oplus P_{3} \oplus S_{2} \oplus S_{3} \oplus M_{2} \rightarrow 0,
$$

where $P_{1} \oplus P_{2} \oplus P_{3} \oplus S_{2} \oplus S_{3} \oplus M_{2}$ is in degree zero, we get $\operatorname{gl} \operatorname{dim}(\Gamma) \leq 3$, but $\operatorname{gl} \operatorname{dim}(\Lambda)<\infty$.

Recall that an Artin algebra $\Lambda$ is called an $F$-Gorenstein algebra if $I^{\infty}(F)=\mathcal{P}^{\infty}(F)$, where $\mathcal{P}^{\infty}(F)=$ $\left\{X \mid\right.$ proj.dim $\left.\operatorname{dim}_{F}(X)<\infty\right\}, I^{\infty}(F)=\left\{Y \mid \operatorname{inj} \cdot \operatorname{dim}_{F}(Y)<\infty\right\}$. 
Lemma 7.7. 5. Lemma 3.2] An Artin algebra $\Lambda$ is an $F$-Gorenstein algebra if and only if both the relative injective dimension of all modules in $\mathcal{P}(F)$ and the relative projective dimension of all modules in $I(F)$ are bounded.

It is well known that Gorensteinness is invariant under derived equivalence. It is not immediately obvious that the relation between $F$-Gorensteinness and relative derived equivalences, but this will follow from the following propositions.

Proposition 7.8. Let $L: \mathrm{D}_{\mathrm{F}}^{\mathrm{b}}(\Lambda) \rightarrow \mathrm{D}^{\mathrm{b}}(\Gamma)$ be a relative derived equivalence. Suppose that $T^{\bullet}$ is the relative tilting complex associated to $L$. Then inj. $\operatorname{dim}_{F}(\mathcal{P}(F))-t\left(T^{\bullet}\right) \leq \mathrm{inj} \cdot \operatorname{dim}\left({ }_{\Gamma} \Gamma\right) \leq \operatorname{inj} \cdot \operatorname{dim}_{F}(\mathcal{P}(F))+$ $t\left(T^{\bullet}\right)+2$.

Proof. Assume that inj. $\operatorname{dim}_{F}(\mathcal{P}(F))<\infty$. Set $r=\max \{\operatorname{inj} \cdot \operatorname{dim}(P) \mid P \in \mathcal{P}(F)\}$. By homological formula, we have

$$
\operatorname{Ext}_{\Gamma}^{i}(X, \Gamma) \simeq \operatorname{Hom}_{D^{b}(\Gamma)}(X, \Gamma[i]) \simeq \operatorname{Hom}_{D_{\mathrm{F}}^{\mathrm{b}}(\Lambda)}\left(H(X), T^{\bullet}[i]\right) .
$$

By Lemma 7.4 $H(X)$ is isomorphic in $\mathrm{D}_{\mathrm{F}}^{\mathrm{b}}(\Lambda)$ to a complex of the form:

$$
\cdots \rightarrow 0 \rightarrow \operatorname{Im}\left(d_{T_{X}}^{-n-2}\right) \rightarrow T_{X}^{-n-1} \rightarrow \cdots \rightarrow T_{X}^{-1} \rightarrow \operatorname{Ker}\left(d_{T_{X}}^{0}\right) \rightarrow 0 \rightarrow \cdots .
$$

Moreover, $T^{\bullet} \in \mathrm{K}^{\mathrm{b}}(\mathcal{P}(F))$ and inj. $\operatorname{dim}_{F}(\mathcal{P}(F))<\infty$. By Lemma 7.1, it follows that $\operatorname{Ext}_{F}^{i}(-, P)=0$ for $i \geq r+1$. By Lemma 7.6 we deduce that $\operatorname{Ext}_{\Gamma}^{i}(X, \Gamma)=0$ for $i \geq r+1+n+2$. Then inj.dim $(\Gamma \Gamma) \leq$ $n+2+\operatorname{inj} \cdot \operatorname{dim}_{F}(\mathcal{P}(F))$.

Suppose that inj.dim $\left({ }_{\Gamma} \Gamma\right)<\infty$. By homological formula, we have

$$
\operatorname{Ext}_{F}^{i}(Y, P) \simeq \operatorname{Hom}_{\mathrm{D}_{\mathrm{F}}^{\mathrm{b}}(\Lambda)}(Y, P[i]) \simeq \operatorname{Hom}_{\mathrm{D}^{\mathrm{b}}(\Gamma)}(L(Y), L(P)[i]) .
$$

By Lemma7.5, $L(Y)$ is isomorphic in $\mathrm{D}^{\mathrm{b}}(\Gamma)$ to a complex of the form:

$$
\cdots \rightarrow 0 \rightarrow \operatorname{Coker}\left(d_{T_{Y}}^{-1}\right) \rightarrow \bar{T}_{Y}^{0} \rightarrow \cdots \rightarrow \bar{T}_{Y}^{n-1} \rightarrow \operatorname{Ker}\left(d_{T_{Y}}^{n}\right) \rightarrow 0 \rightarrow \cdots
$$

By Lemma 7.6, we conclude that $\operatorname{Ext}_{F}^{i}(Y, P)=0$ for $i \geq i n j \cdot \operatorname{dim}\left({ }_{\Gamma} \Gamma\right)+1+n$. Consequently, inj. $\operatorname{dim}_{F}(\mathcal{P}(F)) \leq$ inj.dim $\left({ }_{\Gamma} \Gamma\right)+n$. This completes the proof.

Proposition 7.9. Let $\Lambda$ and $\Gamma$ be relatively derived equivalent Artin $R$-algebras. Then $\Lambda$ is $F$-Gorenstein if and only if $\Gamma$ is Gorenstein.

Proof. Assume that $\Lambda$ is an $F$-Gorenstein algebra. Then we have inj. $\operatorname{dim}_{F}(\mathcal{P}(F))<\infty$ and proj. $\operatorname{dim}_{F}(I(F))<\infty$. Set $r=\max \left\{\right.$ proj.dim $\left.\operatorname{dim}_{F}(J) \mid J \in I(F)\right\}$. For any injective $\Gamma$-module $I$, we have

$$
\operatorname{Ext}_{\Gamma}^{i}(I, X) \simeq \operatorname{Hom}_{\mathrm{D}^{\mathrm{b}}(\Gamma)}(I, X[i]) \simeq \operatorname{Hom}_{\mathrm{D}_{\mathrm{F}}^{\mathrm{b}}(\Lambda)}(H(I), H(X)[i]) .
$$

By Lemmas 6.5 and 7.4, we conclude that $H(I) \in \mathrm{K}^{\mathrm{b}}(I(F))$ and $H(I)$ is isomorphic in $\mathrm{D}_{\mathrm{F}}^{\mathrm{b}}(\Lambda)$ to a complex $J^{\bullet}$ of the following form:

$$
\cdots \rightarrow 0 \rightarrow \operatorname{Im}\left(d_{J}^{-n-2}\right) \rightarrow J^{-n-1} \rightarrow \cdots \rightarrow J^{-1} \rightarrow J^{0} \rightarrow \cdots \rightarrow J^{s} \rightarrow 0
$$

such that $\operatorname{Im}\left(d_{J}^{-n-2}\right), J^{-i}$ are $F$-injective $\Lambda$-modules for $-n-1 \leq i \leq s$. By Lemma 7.6, we see that $\operatorname{Ext}_{\Gamma}^{i}(I, X)=0$ for $i \geq r+1+n+2$. This implies that proj.dim $(I) \leq n+2+\operatorname{proj}^{-d i m} \operatorname{dim}_{F}(I(F))$. Therefore, inj.dim $\left(\Gamma_{\Gamma}\right)<\infty$. By Proposition 7.8, it follows that if inj. $\operatorname{dim}_{F}(\mathcal{P}(F))<\infty$, then inj.dim $\left(\Gamma_{\Gamma} \Gamma\right)<\infty$. Therefore, $\Gamma$ is a Gorenstein algebra.

If $\Gamma$ is a Gorenstein algebra, then inj. $\operatorname{dim}\left({ }_{\Gamma} \Gamma\right)=\operatorname{inj} \cdot \operatorname{dim}\left(\Gamma_{\Gamma}\right)=s<\infty$. In the same way, we deduce that $\Lambda$ is an $F$-Gorenstein algebra. This completes the proof.

In particular, when $F=\operatorname{Ext}_{\Lambda}^{1}(-,-)$, by the proof of Theorem 7.3, we have the following.

Corollary 7.10. Let $\Lambda$ and $\Gamma$ be derived equivalent. Suppose $T^{\bullet}$ is a tilting complex for $\Lambda$. Then

(1) gl.dim $(\Lambda)-l\left(T^{\bullet}\right) \leq$ gl.dim $(\Gamma) \leq$ gl.dim $(\Lambda)+l\left(T^{\bullet}\right)$.

(2) fin.dim $(\Lambda)-l\left(T^{\bullet}\right) \leq$ fin.dim $(\Gamma) \leq$ fin.dim $(\Lambda)+l\left(T^{\bullet}\right)$.

(3) inj.dim $(\Lambda)-l\left(T^{\bullet}\right) \leq$ inj.dim $(\Gamma) \leq$ inj.dim $(\Lambda)+l\left(T^{\bullet}\right)$. 
Acknowledgements. The author gratefully thanks Professor Changchang Xi for his many helpful suggestions and would like to acknowledge National Natural Science Foundation of China (No. 11201022) and the Fundamental Research Funds for the Central Universities (2013JBM096, 2013RC027). The author also would like to thank the referee for his/her helpful comments that improve the paper.

\section{References}

[1] Auslander, M., Reiten, I., Smal Ø, S. O.: Representation Theory of Artin Algebras. Cambridge University Press, 1995.

[2] Auslander, M., Solberg, Ø.: Relative homology and representation theory I, Relative homology and homologically finite subcategories. Comm. Algebra 21(1993), 2995-3031.

[3] Auslander, M., Solberg, Ø.: Relaive homology and representation theory II, Relative cotilting theory. Comm. Algebra 21(1993), 3033-3079.

[4] Auslander, M., Solberg, Ø.: Relative homology and representation theory III, Cotilting modules and Wedderburn correspondence. Comm. Algebra 21(1993), 3081-3097.

[5] Auslander, M., Solberg, Ø.: Gorenstein algebras and algebras with dominant dimension at least 2. Comm. Algebra 21(1993), 3897-3934.

[6] Auslander, M., Solberg, Ø.: Relative homology. Finite-dimensional algebras and related topics (Ottawa, ON, 1992), 347-359, NATO Adv. Sci. Inst. Ser. C Math. Phys. Sci., 424, Kluwer Acad. Publ., Dordrecht, 1994.

[7] BuAn, A.: Closed subbifunctors of the extension bifunctor. J. Algebra 244(2001), 407-428.

[8] BUAn, A.: Subcategories of the derived category and cotilting complexes. Colloq. Math. 88(2001), $1-11$.

[9] Buchweitz, R. O.: Maximal Cohen-Macaulay modules and Tate-cohomology over Gorenstein rings, Hamburg, p. 155 (1987). (unpublished manuscript)

[10] Chen, X. W.: Gorenstein homological algebra of Artin algebras, Postdoctoral Report, USTC, 2010.

[11] Chen, X. W., Ye, Y., Zhang, P.: Algebras of dereived dimension of zero. Comm. Algebra 36(2008), 1-10.

[12] Chen, X. W., Zhang, P.: Quotient triangulated categories. Manuscripta Math. 123(2007), 167183.

[13] Cline, E., Parshall, B., Scott L.: Derived categories and Morita theory. J. Algebra 104(1986), 397-409.

[14] P. Draxler, I. Reiten, S. O. Smal $\varnothing$ and $\varnothing$. Solberg, Exact categories and vector space categories. Trans. Amer. Math. Soc. 351(1999), 647-682.

[15] Dugger, D., Shipley, B.: K-theory and derived equivalences. Duke Math. J. 124(2004), 587-617.

[16] Enochs, E. E., Jenda, O. M. G.: Relative Homological Algebra. De Gruyter Expositions in Math. 30, Walter de Gruyter-Berlin-New York, 2000.

[17] GAO, N., ZhANG, P.: Gorenstein derived categories. J. Algebra 323(2010), 2041-2057.

[18] Gelfand, S. I., Manin, Y.: Methods of Homological Algebra. Springer Monographs in Math. (2nd ed.), Springer-Verlag, 2002.

[19] Generalov, A. I.: Relative homological algebra. Cohomology of categories, posets and coalgebras, in "Handbook of Algebra" V.1, 611-638. North-Holland, Amsterdam, 1996.

[20] Grime, M.: Adjoint functors and triangulated categories. Comm. Algebra 36(2008), 3589-3607.

[21] Happel, D.: Triangulated Categories in the Representation Theory of Finite Dimensional Algebras. Cambridge University Press, Cambridge. 1988.

[22] Happel, D.: On the derived category of finite-dimensional algebra. Comment. Math. Helv. 62(1987), 339-389. 
[23] HAPPEL, D.: On Gorenstein algebras. In: Representation theory of finite groups and finitedimensional algebras (Proc. Conf. at Bielefeld, 1991), 389-404, Progress in Math., vol. 95, Birkhäuser, Basel, 1991.

[24] Harshorne, R.: Duality and Residue. Lecture Notes in Math. 20, Springer, Berlin, 1966.

[25] HochsChILD, G.: Relative homological algebra. Trans. Amer. Math. Soc. 82(1956), 246-269.

[26] Hoshino, M., Kato, Y.: Tilting complexes defined by idempotents. Comm. Algebra 30(2002), 83-100.

[27] Hoshino, M., Kato, Y.: Tilting complexes associated with a sequence of idempotents. J. Algebra 183(2003), 105-124.

[28] Hu, W., XI, C. C.: D-split sequences and derived equivalences. Adv. Math. 227(2011), 292-318.

[29] Hu, W., XI, C. C.: Derived equivalences and stable equivalences of Morita type, I. Nagoya Math. J. $200(2010), 107-152$.

[30] HU, W., XI, C. C.: Derived equivalences for $\Phi$-Auslander-Yoneda algebras. Trans. Amer. Math. Soc. 365(2013), 5681-5711.

[31] Kashiwara, M., Schapira, P.: Sheaves on Manifolds. Grundlehren der mathematischen Wissenschaften 292 Berlin: Springer-Verlag, 1990.

[32] Kato, Y.: On derived equivalent coherent rings. Comm. Algebra 30(2002), 4437-4454.

[33] Keller, B.: Deriving DG categories. Ann. Sci. école Norm. Sup. 27 (1994), 63-102.

[34] Konig, S.,Zimmermann, A.: Derived Equivalences for Group Rings. Lecture notes in math. 1685, Berlin: Springer-Verlag, 1998

[35] Krause, H.: Localization theory for triangulated categories. To appear in the proceedings of the "Workshop on Triangulated Categories" in Leeds, 2006.

[36] MiyashitA, Y.: Tilting modules associated with a series of idempotent ideals. J. Algebra 238 (2001), 485C501.

[37] Neeman, A. Triangulated Categories. Annals of Mathematics Studies 148, Princeton University Press, Princeton and Oxford, 2001.

[38] PAN, S. Y.: Relative quotient triangulated categories. Accepted by Algebra Colloquium.

[39] PAN, S. Y., XI, C. C.: Finiteness of finitistic dimension is invariant under derived equivalences. $J$. Algebra 322(2009), 21-24.

[40] Rickard, J.: Morita theory for derived categories. J. London Math. Soc. 39 (1989), 436-456.

[41] Rickard, J.: Derived categories and stable equivalence. J. Pure Appl. Algebra 61 (1989), 303-317.

[42] Rickard, J.: Derived equivalences as derived functors. J. London Math. Soc. 43 (1991), 37-48.

[43] Rotman, J.: An Introduction to Homological Algebra. Academic Press New York, San Francisco, London, 1979.

[44] Thomason, R. W., Trobaugh, T. F.: Higher algebraic $K$-theory of schemes and of derived categories. Then Grothendieck Festschrift (a collection of papers to honor Grothendieck's 60th birthday) vol 3, Birkhäuser, 1990, pp. 247-435.

[45] Verdier, J.: Catégories dérivées, état 0. Lecture Notes in Math. 569(1977), Springer, Berlin, 262-311.

[46] XI, C. C.: The relative Auslander-Reiten theory of modules. Preprint, available at : http://math.bnu.edu.cn/ ccxi/Papers/Articles/rart.pdf, 2002.

[47] Weibel, C. A.: An Introduction to Homological Algebra. Cambridge Studies in Advanced Math. 38, Cambridge University Press, 1994. 